Supplementary material 1: Analyses of the concentrations of macro-nutrients (nitrate, $\mathrm{NO}_{3}$; nitrite, $\mathrm{NO}_{2}$; and silicic acid, $\left.\mathrm{Si}(\mathrm{OH})_{4}\right)$, biogenic silica (BSi), and particulate organic carbon and nitrogen (POC and PON).

\title{
1. Sampling:
}

The GEOVIDE cruise was conducted in May-June 2014 and consisted in 78 stations. Stations were labeled as Short (46), Large (17), XLarge (5), and Super (10) stations depending on the number of parameters and rosette casts. The first station cast was used as reference for physicochemical characterization of water masses. During the first cast at each station, a standard stainless steel rosette (SSSR) equipped with 22 Niskin bottles and a CTD (SBE911 and SBE9+) equipped with a SBE-43 was deployed.

For the analyses of macro-nutrients, samples were taken in all the 78 stations from the SSSR. For BSi and POC/PON measurements, samples were collected from the SSSR at all Large, XLarge and Super stations.

\section{Analytical procedures}

\subsection{Macro-nutrients}

\subsubsection{Apparatus and analysis protocols}

Analyses for nitrite and nitrate concentrations were conducted on unfiltered samples directly aboard the R.V. Pourquoi Pas?. Unfiltered samples were stored in $15 \mathrm{~mL}$ plastic tubes at $+5^{\circ} \mathrm{C}$, for analyses of silicic acid concentrations at the LEMAR laboratory.

For the measurements of the macro-nutrients concentrations in the GEOVIDE samples, a SEAL AutoAnalyzer II continuous flow analyzer (CFA) were used aboard the R.V. Pourquoi Pas?, and a SEAL AutoAnalyzer III at the LEMAR laboratory in Brest, following the protocols described by Aminot \& Kerouel (2007) and using very high purity reagents. Deionized ("Millipore") water was run through the CFA to determine the optical base line of the CFA for the determination of nitrite, nitrate, and silicic acid concentration.

Typically, the analysis of the samples of a given station was performed successively, running through the CFA: (i) a set of subsamples of standard solutions (cf. 2.1.2.), (ii) duplicates of samples of the station, and (iii) a set of subsamples of standard solutions and then 5- to correct from CFA refraction signal, all the samples were then re-run through the CFA channels, according to Aminot and Kerouel (2007).

For the GEOVIDE cruise, the repeatability between samples was better than $0.003 \mu \mathrm{M}$ for nitrite, better than $0.02 \mu \mathrm{M}$ for nitrate, and better than $0.02 \mu \mathrm{M}$ for silicic acid.

\subsubsection{Calibration procedures}

According to Aminot and Kerouel (2007) artificial seawater was used for the preparation of calibration standards. All the volumetric glass- and plastic ware used were gravimetrically calibrated at the temperature of the laboratory (about $20^{\circ} \mathrm{C}$ ). All volumetric flasks used were Class A+. All pipets used have a nominal calibration tolerance of $0.1 \%$ or better. 
Eight seawater standards were made for each parameters: For nitrite, between 0 and $2 \mu \mathrm{M}$; for nitrate, between 0 and $25 \mu \mathrm{M}$; and for silicic acid between 0 and $60 \mu \mathrm{M}$ for deep stations and between 0 and $25 \mu \mathrm{M}$ for shallow stations.

Calibration equations was strictly linear for nitrite and nitrate. Calibration was second order polynomial for silicic acid in the $0-60 \mu \mathrm{M}$ range.

\subsubsection{Certification of the measurements}

To certify the GEOVIDE primary standard solutions of nitrite, nitrate, and silicic acid used aboard the R.V. Pourquoi Pas?, triplicates of KANSO international standards (refs: CA, CD, BZ and BW certified standards; KANSO, the general Environmental Technos Co., Ldt, Laboratory for Instrumentation analysis, 3-1-1, Higashikuraji, Katano, Osaka, 576-0061 Japan) were analyzed using the LEMAR CFA analyzer.

Table 1. Concentrations of KANSO standard solutions measured using the LEMAR AutoAnalyzer III compared to KANSO certified concentrations $(\mu \mathrm{M})$.

\begin{tabular}{lllll}
\hline & \multicolumn{2}{l}{ KANSO measured } & \multicolumn{2}{l}{ KANSO certified } \\
& Concentrations & Uncertainty & Concentrations & Uncertainty \\
\hline Nitrite & 0.028 & 0.001 & 0.018 & 0.004 \\
& 0.071 & 0.001 & 0.065 & 0.010 \\
& 0.075 & 0.001 & 0.069 & 0.010 \\
Nitrate & & & & \\
& 5.45 & 0.01 & 5.63 & 0.05 \\
& 19.48 & 0.04 & 20.13 & 0.15 \\
Silicic acid & 24.4 & 0.04 & 25.18 & 0.20 \\
& 14.23 & 0.03 & & \\
& 39.96 & 0.03 & 14.26 & 0.10 \\
& 60.31 & 0.03 & 61.46 & 0.23 \\
& & & & 0.43 \\
\hline
\end{tabular}

Table 1 shows that, in the range of the concentrations measured during the GEOVIDE cruise (nitrite: 0-1 $\mu \mathrm{M}$; nitrate: 0-25 $\mu \mathrm{M}$; silicic acid 0-60 $\mu \mathrm{M}$ ), the KANSO measured concentrations were significantly different from the KANSO certified values. Plotting measured vs. certified concentrations of nitrite, nitrate and silicic acid given in Table 1, linear regression equations were calculated. The measured concentrations of the GEOVIDE samples were corrected, consequently.

\subsubsection{Accuracy of the GEOVIDE measurements:}

Concentrations of nutrients $\left(\mu \mathrm{mol} \mathrm{L}^{-1}\right.$ ) are given with an accuracy of \pm 0.01 for nitrite, \pm 0.1 for nitrate and \pm 0.2 silicic acid.

Nominally, the accuracy of the silicic aid concentrations should be $\pm 0.1 \mu \mathrm{mol} \mathrm{L} \mathrm{L}^{-1}$. However, given the potential interference of biogenic silica in unfiltered samples (concentrations $<0.1$ $\mu \mathrm{M}$ in the deep and bottom waters), we estimated the accuracy to be $\pm 0.2 \mu \mathrm{M}$ for silicic acid. 


\subsection{Biogenic Silica}

Biogenic silica (BSi) was determined from $2.3 \mathrm{~L}$ seawater samples filtered onto polycarbonate filters $(0.6 \mu \mathrm{m}$ pore size, diameter $47 \mathrm{~mm}$ ) immediately after sampling. Filters were dried at $60{ }^{\circ} \mathrm{C}$ and stored for further analysis. BSi concentrations were analysed following Ragueneau et al. (2005). Briefly, a double wet-alkaline digestion is carried out where the filter sample is submitted to a first digestion $\left(0.2 \mathrm{M} \mathrm{NaOH}, \mathrm{pH} \mathrm{13.3)}\right.$ at $100{ }^{\circ} \mathrm{C}$ for $40 \mathrm{~min}$. At the end of this first leach, all the BSi and part of the $\mathrm{LSi}$ have been converted into $\mathrm{Si}(\mathrm{OH})_{4}$. Si and $\mathrm{Al}$ concentrations ([Si $]_{1}$ and $[\mathrm{Al}]_{1}$ ) in the supernatant are analyzed. After rinsing and drying, the filter is submitted to a second digestion, exactly identical to the first one, leading to the determination of the $(\mathrm{Si}: \mathrm{Al})_{2}$ ratio that is characteristic of the silicate minerals present in the sample. The corrected BSi concentration is thus given by:

$[\mathrm{BSi}]=[\mathrm{Si}]_{1}-[\mathrm{Al}]_{1} \times(\mathrm{Si}: \mathrm{Al})_{2}$.

The detection limit is lower than $1.5 \mathrm{nmol}$ of BSi present on the filter and the uncertainty is close to $10 \%$.

\subsection{Particulate organic carbon and nitrogen}

Samples for concentrations of POC and PON were determined from $1.2 \mathrm{~L}$ seawater samples filtered onto pre-combusted glass fibre filters (Whatman GF/F, $25 \mathrm{~mm}$ diameter), rinsed with deionized, distilled water to remove salts, and then dried at $60^{\circ} \mathrm{C}$.

POC and PON samples were analysed after fuming of the filter with concentrated $\mathrm{HCl}$ for $4 \mathrm{~h}$ in a dessicator and drying at $60^{\circ} \mathrm{C}$ in an oven (decarbonation step) (Lorrain et al., 2003). POC and PON were measured using a Flash 2000 Organic Elemental Analyzer Thermo Scientific.

\section{References}

Aminot, A. and Kérouel, R.: Dosage automatique des nutriments dans les eaux marines: méthodes en flux continu, Editions Quae., 2007.

Lorrain, A., Savoye, N., Chauvaud, L., Paulet, Y.-M. and Naulet, N.: Decarbonation and preservation method for the analysis of organic $\mathrm{C}$ and $\mathrm{N}$ contents and stable isotope ratios of low-carbonated suspended particulate material, Anal Chim Acta, 491(2), 125-133, 2003.

Ragueneau, O., Savoye, N., Del Amo, Y., Cotten, J., Tardiveau, B. and Lenaert, A.: A new method for the measurement of biogenic silica in suspended matter of coastal waters: using Si:Al ratios to correct for the mineral interference, Cont Shelf Res, 25, 697-710, 2005. 
Supplementary material 2: Concentrations of macro-nutrients (nitrate, $\mathrm{NO}_{3}$; nitrite, $\mathrm{NO}_{2}$; and silicic acid, $\left.\mathrm{Si}(\mathrm{OH})_{4}\right)$, biogenic silica (BSi), and particulate organic carbon and nitrogen (POC and PON) during the GOEVIDE cruise

\begin{tabular}{|c|c|c|c|c|c|c|c|c|c|c|c|}
\hline Station \# & Cast \# & Latitude $\left({ }^{\circ} \mathrm{N}\right)$ & Longitude $\left({ }^{\circ} \mathrm{E}\right)$ & Date (dd/mm/yy) & Pressure (dbar) & $\mathrm{NO}_{3}\left(\mu \mathrm{mol} \mathrm{L}^{-1}\right)$ & $\mathrm{NO}_{2}\left(\mu \mathrm{mol} \mathrm{L} \mathrm{L}^{-1}\right)$ & $\mathrm{Si}(\mathrm{OH})_{4}\left(\mu \mathrm{mol} \mathrm{L}{ }^{-1}\right)$ & $\mathrm{BSi}\left(\mu \mathrm{mol} \mathrm{L}{ }^{-1}\right)$ & PON $\left(\mu \mathrm{mol} \mathrm{L}^{-1}\right)$ & POC $\left(\mu \mathrm{mol} \mathrm{L}^{-1}\right)$ \\
\hline 1 & 1 & 40.333 & -10.036 & $18 / 05 / 2014$ & 3581 & 19.15 & 0.00 & 41.72 & 0.02 & & \\
\hline & & & & & 3250 & 18.86 & 0.00 & 39.21 & & & \\
\hline & & & & & 3000 & 18.49 & 0.00 & 36.05 & 0.01 & & \\
\hline & & & & & 2500 & 17.69 & 0.00 & 28.61 & 0.01 & & \\
\hline & & & & & 2001 & 16.49 & 0.00 & 18.87 & 0.01 & & \\
\hline & & & & & 1800 & 16.04 & 0.00 & 15.19 & & & \\
\hline & & & & & 1600 & 15.62 & 0.00 & 13.20 & & & \\
\hline & & & & & 1401 & 14.98 & 0.00 & 11.02 & & & \\
\hline & & & & & 1201 & 14.30 & 0.00 & 9.67 & & & \\
\hline & & & & & 1001 & 13.93 & 0.00 & 8.81 & 0.02 & & \\
\hline & & & & & 901 & 13.77 & 0.00 & 8.45 & & & \\
\hline & & & & & 800 & 13.54 & 0.00 & 7.95 & & & \\
\hline & & & & & 701 & 13.48 & 0.00 & 7.42 & & & \\
\hline & & & & & 600 & 13.77 & 0.00 & 7.28 & & & \\
\hline & & & & & 501 & 13.28 & 0.00 & 6.34 & 0.03 & & \\
\hline & & & & & 402 & 11.55 & 0.00 & 4.69 & & & \\
\hline & & & & & 300 & 9.95 & 0.00 & 3.58 & 0.04 & & \\
\hline & & & & & 201 & 7.84 & 0.00 & 2.72 & 0.05 & & \\
\hline & & & & & 149 & 6.78 & 0.00 & 2.17 & & & \\
\hline & & & & & 99 & 6.02 & 0.00 & 1.88 & 0.06 & & \\
\hline & & & & & 60 & 4.54 & 0.00 & 1.09 & 0.09 & & \\
\hline & & & & & 37 & 0.97 & 0.00 & 0.55 & & & \\
\hline & & & & & 16 & 0.04 & 0.00 & 0.87 & 0.06 & & \\
\hline & & & & & 3 & 0.05 & 0.00 & 0.90 & 0.05 & & \\
\hline 1 & 3 & 40.333 & -10.036 & $19 / 05 / 2014$ & 202 & & & & & 0.17 & 1.24 \\
\hline & & & & & 170 & & & & & 0.15 & 1.35 \\
\hline & & & & & 142 & & & & & 0.16 & 0.99 \\
\hline & & & & & 110 & & & & & 0.20 & 2.00 \\
\hline & & & & & 84 & & & & & 0.32 & 2.01 \\
\hline & & & & & 65 & & & & & 0.54 & 3.30 \\
\hline & & & & & 48 & & & & & 1.33 & 6.82 \\
\hline & & & & & 48 & & & & & & \\
\hline & & & & & 48 & & & & & & \\
\hline & & & & & 34 & & & & & 0.88 & 5.29 \\
\hline & & & & & 34 & & & & & & \\
\hline & & & & & 34 & & & & & & \\
\hline & & & & & 25 & & & & & 0.79 & 5.05 \\
\hline & & & & & 25 & & & & & & \\
\hline & & & & & 25 & & & & & & \\
\hline & & & & & 17 & & & & & 0.82 & 5.00 \\
\hline & & & & & 17 & & & & & & \\
\hline & & & & & 17 & & & & & & \\
\hline & & & & & 11 & & & & & 0.81 & 4.85 \\
\hline & & & & & 11 & & & & & & \\
\hline & & & & & 11 & & & & & & \\
\hline & & & & & 6 & & & & & 0.72 & 4.76 \\
\hline & & & & & 6 & & & & & & \\
\hline & & & & & 6 & & & & & & \\
\hline 2 & 1 & 40.333 & -9.459 & $20 / 05 / 2014$ & 142 & 8.25 & 0.06 & 3.73 & 0.11 & & \\
\hline & & & & & 142 & & & & & 0.50 & 4.70 \\
\hline & & & & & 142 & & & & & & \\
\hline & & & & & 121 & & & & 0.07 & 0.20 & 1.39 \\
\hline & & & & & 99 & 5.85 & 0.03 & 1.49 & 0.06 & & \\
\hline & & & & & 99 & & & & & 0.20 & 1.71 \\
\hline & & & & & 86 & & & & & & \\
\hline & & & & & 85 & & & & 0.08 & & \\
\hline & & & & & 77 & & & & & 0.25 & 2.40 \\
\hline & & & & & 39 & & & & & & \\
\hline & & & & & 40 & & & & & & \\
\hline & & & & & 34 & 1.06 & 0.12 & 0.37 & 0.08 & & \\
\hline & & & & & 34 & & & & & 1.10 & 6.72 \\
\hline & & & & & 31 & & & & & & \\
\hline & & & & & 31 & & & & & & \\
\hline & & & & & 26 & & & & 0.07 & 0.97 & 6.44 \\
\hline & & & & & 11 & 0.05 & 0.02 & 0.93 & 0.03 & & \\
\hline & & & & & 11 & & & & & 0.69 & 5.08 \\
\hline & & & & & 11 & & & & & & \\
\hline & & & & & 11 & & & & & & \\
\hline & & & & & 7 & & & & 0.06 & & \\
\hline & & & & & 7 & & & & & & \\
\hline & & & & & 6 & & & & & & \\
\hline & & & & & 7 & & & & & 0.63 & 5.45 \\
\hline 3 & 1 & 40.333 & -9.643 & $21 / 05 / 2014$ & 426 & 13.19 & 0.05 & 5.02 & & & \\
\hline & & & & & 300 & 11.63 & 0.04 & 3.93 & & & \\
\hline & & & & & 201 & 9.11 & 0.04 & 2.72 & & & \\
\hline & & & & & 150 & 7.19 & 0.04 & 2.09 & & & \\
\hline & & & & & 99 & 6.12 & 0.04 & 1.69 & & & \\
\hline & & & & & 60 & 4.48 & 0.13 & 1.07 & & & \\
\hline & & & & & 4 & 0.10 & 0.03 & 1.09 & & & \\
\hline 4 & 1 & 40.333 & -9.766 & $21 / 05 / 2014$ & 808 & 15.24 & 0.01 & 7.70 & & & \\
\hline & & & & & 808 & 15.36 & 0.02 & 7.17 & & & \\
\hline & & & & & 700 & 15.37 & 0.02 & 7.38 & & & \\
\hline & & & & & 700 & 15.49 & 0.02 & 6.87 & & & \\
\hline & & & & & 600 & 14.95 & 0.02 & 5.99 & & & \\
\hline & & & & & 501 & 12.38 & 0.02 & 3.98 & & & \\
\hline & & & & & 400 & 11.36 & 0.02 & 3.43 & & & \\
\hline
\end{tabular}




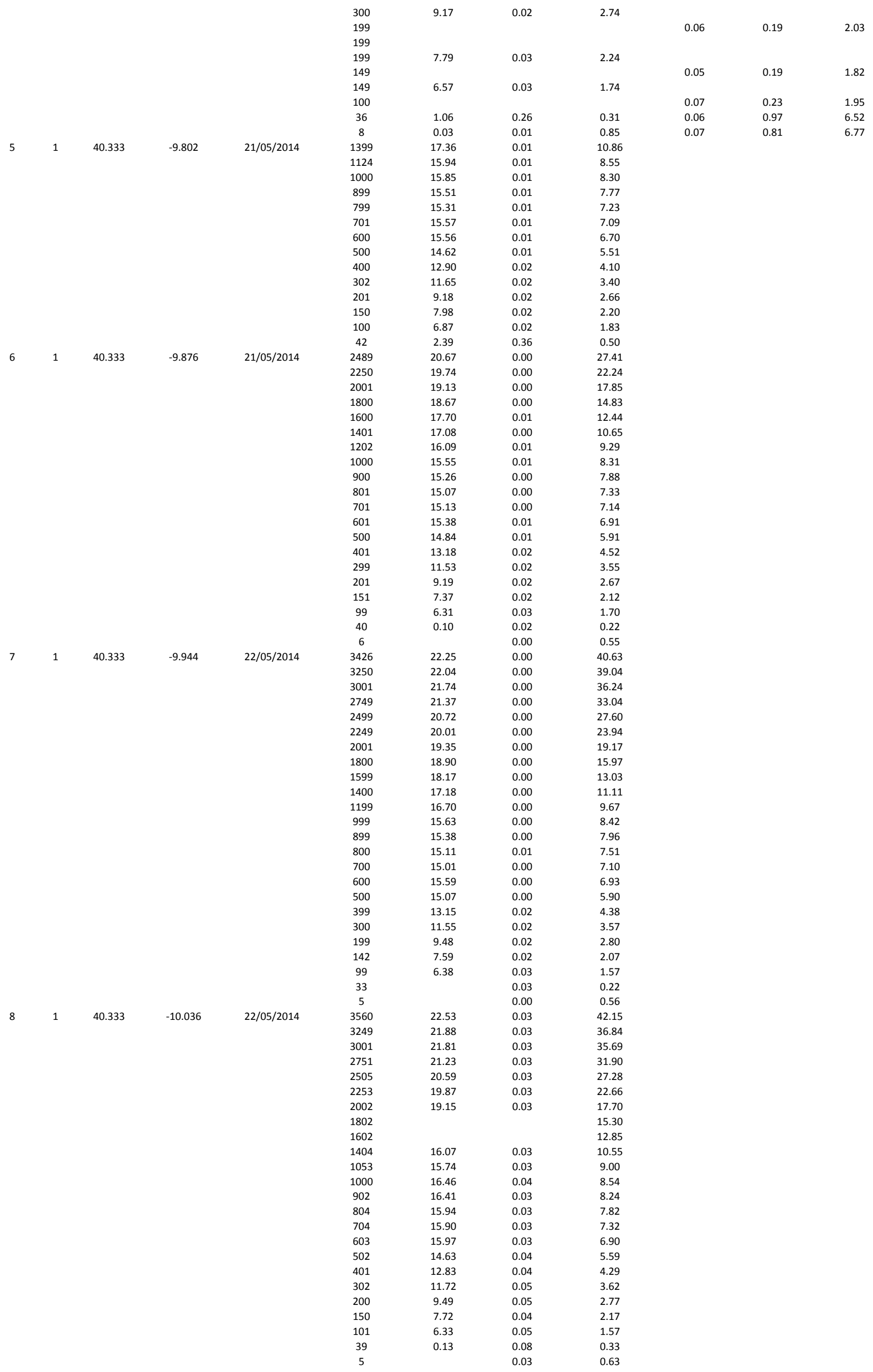




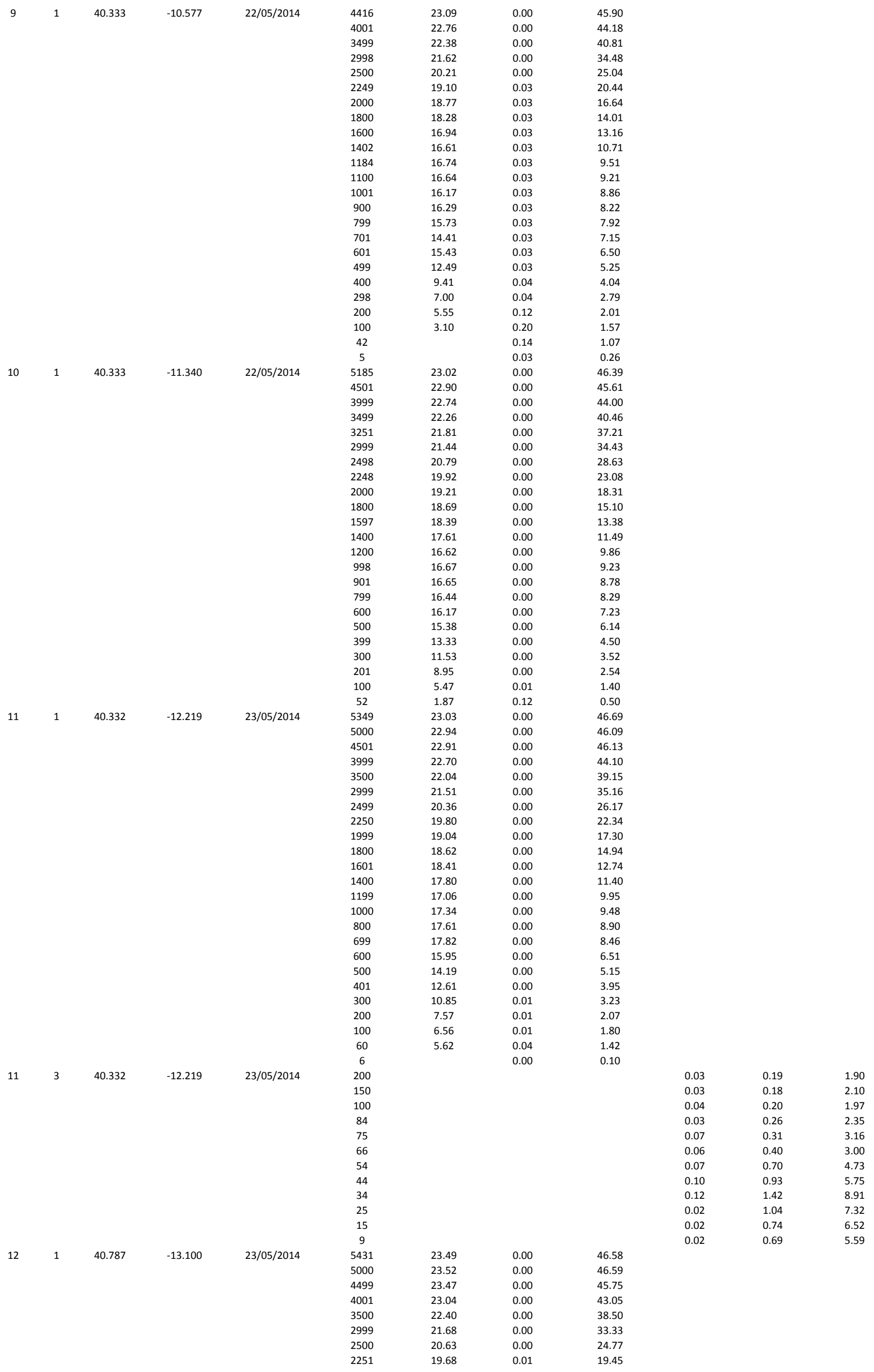




\begin{tabular}{|c|c|c|c|c|c|c|c|}
\hline & & & & & 1999 & 19.16 & 0.01 \\
\hline & & & & & 1800 & 18.99 & 0.01 \\
\hline & & & & & 1601 & 18.77 & 0.01 \\
\hline & & & & & 1399 & 18.19 & 0.01 \\
\hline & & & & & 1200 & 17.34 & 0.02 \\
\hline & & & & & 999 & 17.10 & 0.02 \\
\hline & & & & & 801 & 17.11 & 0.01 \\
\hline & & & & & 699 & 17.54 & 0.02 \\
\hline & & & & & 501 & 12.57 & 0.02 \\
\hline & & & & & 400 & 10.93 & 0.02 \\
\hline & & & & & 301 & 8.85 & 0.01 \\
\hline & & & & & 200 & 7.79 & 0.02 \\
\hline & & & & & 101 & 6.68 & 0.02 \\
\hline & & & & & 66 & 5.66 & 0.04 \\
\hline & & & & & 5 & 0.06 & 0.00 \\
\hline 13 & 1 & 41.383 & -13.888 & $24 / 05 / 2014$ & 5440 & 23.31 & 0.00 \\
\hline & & & & & 4999 & 23.33 & 0.00 \\
\hline & & & & & 4500 & 23.23 & 0.00 \\
\hline & & & & & 4001 & 22.98 & 0.00 \\
\hline & & & & & 3499 & 22.28 & 0.00 \\
\hline & & & & & 3001 & 21.45 & 0.00 \\
\hline & & & & & 2500 & 20.15 & 0.00 \\
\hline & & & & & 2250 & 19.70 & 0.00 \\
\hline & & & & & 2000 & 19.11 & 0.00 \\
\hline & & & & & 1750 & 18.82 & 0.00 \\
\hline & & & & & 1500 & 18.58 & 0.00 \\
\hline & & & & & 1251 & 18.00 & 0.00 \\
\hline & & & & & 1000 & 18.88 & 0.00 \\
\hline & & & & & 799 & 18.36 & 0.00 \\
\hline & & & & & 700 & 17.29 & 0.01 \\
\hline & & & & & 600 & 13.89 & 0.01 \\
\hline & & & & & 499 & 11.62 & 0.00 \\
\hline & & & & & 400 & 9.68 & 0.02 \\
\hline & & & & & 300 & 10.29 & 0.01 \\
\hline & & & & & 201 & 7.85 & 0.02 \\
\hline & & & & & 100 & 6.79 & 0.02 \\
\hline & & & & & 49 & 2.93 & 0.30 \\
\hline & & & & & 30 & 0.70 & 0.06 \\
\hline & & & & & 4 & 0.05 & 0.01 \\
\hline 13 & 3 & 41.383 & -13.888 & $24 / 05 / 2014$ & 180 & & \\
\hline & & & & & 160 & & \\
\hline & & & & & 140 & & \\
\hline & & & & & 116 & & \\
\hline & & & & & 99 & & \\
\hline & & & & & 80 & & \\
\hline & & & & & 58 & & \\
\hline & & & & & 35 & & \\
\hline & & & & & 15 & & \\
\hline & & & & & 10 & & \\
\hline & & & & & 5 & & \\
\hline 14 & 1 & 41.983 & -14.673 & $27 / 05 / 2014$ & 5425 & 23.57 & 0.01 \\
\hline & & & & & 4999 & 23.21 & 0.01 \\
\hline & & & & & 4497 & 23.47 & 0.01 \\
\hline & & & & & 4000 & 23.18 & 0.00 \\
\hline & & & & & 3500 & 22.28 & 0.01 \\
\hline & & & & & 3001 & 21.45 & 0.00 \\
\hline & & & & & 2498 & 20.00 & 0.00 \\
\hline & & & & & 2202 & 19.52 & 0.00 \\
\hline & & & & & 2000 & 19.22 & 0.00 \\
\hline & & & & & 1800 & 18.96 & 0.00 \\
\hline & & & & & 1601 & 18.85 & 0.00 \\
\hline & & & & & 1401 & 18.51 & 0.00 \\
\hline & & & & & 1200 & 17.90 & 0.00 \\
\hline & & & & & 1000 & 17.74 & 0.00 \\
\hline & & & & & 799 & 18.42 & 0.00 \\
\hline & & & & & 700 & 17.31 & 0.01 \\
\hline & & & & & 600 & 14.72 & 0.00 \\
\hline & & & & & 500 & 12.28 & 0.01 \\
\hline & & & & & 400 & 10.91 & 0.01 \\
\hline & & & & & 301 & 9.51 & 0.01 \\
\hline & & & & & 199 & 8.88 & 0.01 \\
\hline & & & & & 100 & 8.37 & 0.02 \\
\hline & & & & & 39 & 3.22 & 0.13 \\
\hline & & & & & 5 & 0.82 & 0.07 \\
\hline 15 & 1 & 42.581 & -15.461 & $28 / 05 / 2014$ & 5148 & 23.50 & 0.00 \\
\hline & & & & & 4999 & 23.39 & 0.00 \\
\hline & & & & & 4500 & 23.42 & 0.00 \\
\hline & & & & & 4000 & 23.18 & 0.00 \\
\hline & & & & & 3499 & 22.41 & 0.00 \\
\hline & & & & & 3000 & 21.85 & 0.00 \\
\hline & & & & & 2500 & 20.35 & 0.00 \\
\hline & & & & & 2250 & 19.32 & 0.00 \\
\hline & & & & & 2000 & 18.87 & 0.00 \\
\hline & & & & & 1799 & 18.93 & 0.00 \\
\hline & & & & & 1599 & 18.65 & 0.00 \\
\hline & & & & & 1399 & 18.59 & 0.01 \\
\hline & & & & & 1199 & 17.99 & 0.00 \\
\hline & & & & & 996 & 18.48 & 0.01 \\
\hline & & & & & 799 & 17.89 & 0.01 \\
\hline & & & & & 700 & 17.44 & 0.01 \\
\hline & & & & & 601 & 15.43 & 0.01 \\
\hline
\end{tabular}




\begin{tabular}{|c|c|c|c|c|c|c|c|}
\hline & & & & & 500 & 13.92 & 0.01 \\
\hline & & & & & 400 & 11.32 & 0.01 \\
\hline & & & & & 300 & 11.45 & 0.00 \\
\hline & & & & & 200 & 8.97 & 0.00 \\
\hline & & & & & 100 & 8.35 & 0.01 \\
\hline & & & & & 51 & 5.44 & 0.62 \\
\hline & & & & & 4 & 0.86 & 0.08 \\
\hline 15 & 3 & 42.581 & -15.461 & $28 / 05 / 2014$ & 204 & & \\
\hline & & & & & 143 & & \\
\hline & & & & & 101 & & \\
\hline & & & & & 80 & & \\
\hline & & & & & 66 & & \\
\hline & & & & & 60 & & \\
\hline & & & & & 50 & & \\
\hline & & & & & 40 & & \\
\hline & & & & & 30 & & \\
\hline & & & & & 20 & & \\
\hline & & & & & 10 & & \\
\hline & & & & & 2 & & \\
\hline 16 & 1 & 43.181 & -16.245 & $28 / 05 / 2014$ & 5212 & 23.28 & 0.03 \\
\hline & & & & & 5000 & 23.27 & 0.01 \\
\hline & & & & & 4498 & 23.29 & 0.00 \\
\hline & & & & & 3999 & 23.06 & 0.00 \\
\hline & & & & & 3495 & 22.34 & 0.00 \\
\hline & & & & & 3000 & 21.29 & 0.00 \\
\hline & & & & & 2500 & 19.69 & 0.02 \\
\hline & & & & & 2250 & 19.05 & 0.00 \\
\hline & & & & & 2000 & 18.68 & 0.01 \\
\hline & & & & & 1800 & 18.61 & 0.00 \\
\hline & & & & & 1600 & 18.65 & 0.01 \\
\hline & & & & & 1401 & 18.65 & 0.01 \\
\hline & & & & & 1200 & 18.36 & 0.00 \\
\hline & & & & & 1001 & 18.15 & 0.00 \\
\hline & & & & & 800 & 17.46 & 0.00 \\
\hline & & & & & 700 & 16.58 & 0.00 \\
\hline & & & & & 600 & 16.10 & 0.00 \\
\hline & & & & & 499 & 13.69 & 0.01 \\
\hline & & & & & 400 & 12.08 & 0.01 \\
\hline & & & & & 301 & 10.32 & 0.00 \\
\hline & & & & & 200 & 9.28 & 0.02 \\
\hline & & & & & 101 & 7.80 & 0.04 \\
\hline & & & & & 40 & 3.40 & 0.00 \\
\hline & & & & & 5 & 0.55 & 0.09 \\
\hline 17 & 1 & 43.780 & -17.032 & $29 / 05 / 2014$ & 4063 & 22.80 & 0.01 \\
\hline & & & & & 3499 & 21.96 & 0.00 \\
\hline & & & & & 3250 & 21.25 & 0.00 \\
\hline & & & & & 2999 & 20.52 & 0.00 \\
\hline & & & & & 2501 & 19.12 & 0.00 \\
\hline & & & & & 2250 & 18.64 & 0.00 \\
\hline & & & & & 1999 & 18.50 & 0.00 \\
\hline & & & & & 1799 & 18.44 & 0.00 \\
\hline & & & & & 1600 & 18.42 & 0.00 \\
\hline & & & & & 1401 & 18.56 & 0.00 \\
\hline & & & & & 1201 & 18.48 & 0.00 \\
\hline & & & & & 1201 & 18.44 & 0.01 \\
\hline & & & & & 998 & 18.52 & 0.00 \\
\hline & & & & & 900 & 18.81 & 0.01 \\
\hline & & & & & 797 & 18.25 & 0.01 \\
\hline & & & & & 699 & 16.52 & 0.01 \\
\hline & & & & & 600 & 15.79 & 0.01 \\
\hline & & & & & 500 & 12.96 & 0.02 \\
\hline & & & & & 401 & 11.18 & 0.01 \\
\hline & & & & & 301 & 10.34 & 0.01 \\
\hline & & & & & 201 & 9.37 & 0.01 \\
\hline & & & & & 101 & 8.42 & 0.02 \\
\hline & & & & & 20 & 1.89 & 0.16 \\
\hline & & & & & 4 & 1.68 & 0.09 \\
\hline 17 & 3 & 43.780 & -17.031 & $29 / 05 / 2014$ & 200 & & \\
\hline & & & & & 150 & & \\
\hline & & & & & 100 & & \\
\hline & & & & & 81 & & \\
\hline & & & & & 69 & & \\
\hline & & & & & 60 & & \\
\hline & & & & & 50 & & \\
\hline & & & & & 40 & & \\
\hline & & & & & 30 & & \\
\hline & & & & & 20 & & \\
\hline & & & & & 10 & & \\
\hline & & & & & 5 & & \\
\hline 18 & 1 & 44.377 & -17.817 & $30 / 05 / 2014$ & 4500 & 23.22 & 0.00 \\
\hline & & & & & 4005 & 23.00 & 0.00 \\
\hline & & & & & 3504 & 21.67 & 0.00 \\
\hline & & & & & 3251 & 21.28 & 0.00 \\
\hline & & & & & 3001 & 20.78 & 0.00 \\
\hline & & & & & 2501 & 19.13 & 0.01 \\
\hline & & & & & 2251 & 19.01 & 0.01 \\
\hline & & & & & 2002 & 18.74 & 0.00 \\
\hline & & & & & 1801 & 18.60 & 0.00 \\
\hline & & & & & 1602 & 18.67 & 0.00 \\
\hline & & & & & 1401 & 18.67 & 0.00 \\
\hline & & & & & 1200 & 18.71 & 0.00 \\
\hline
\end{tabular}




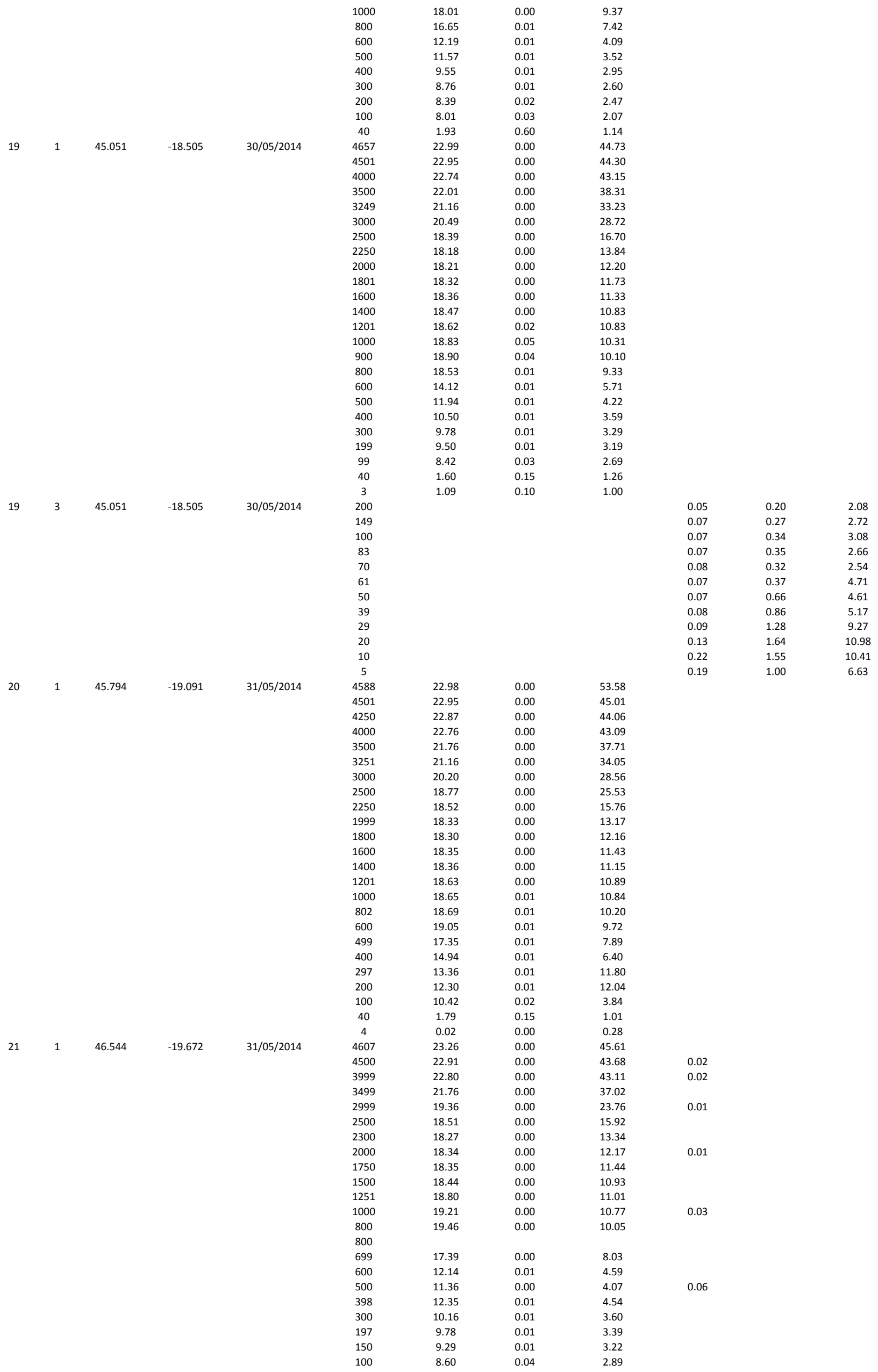




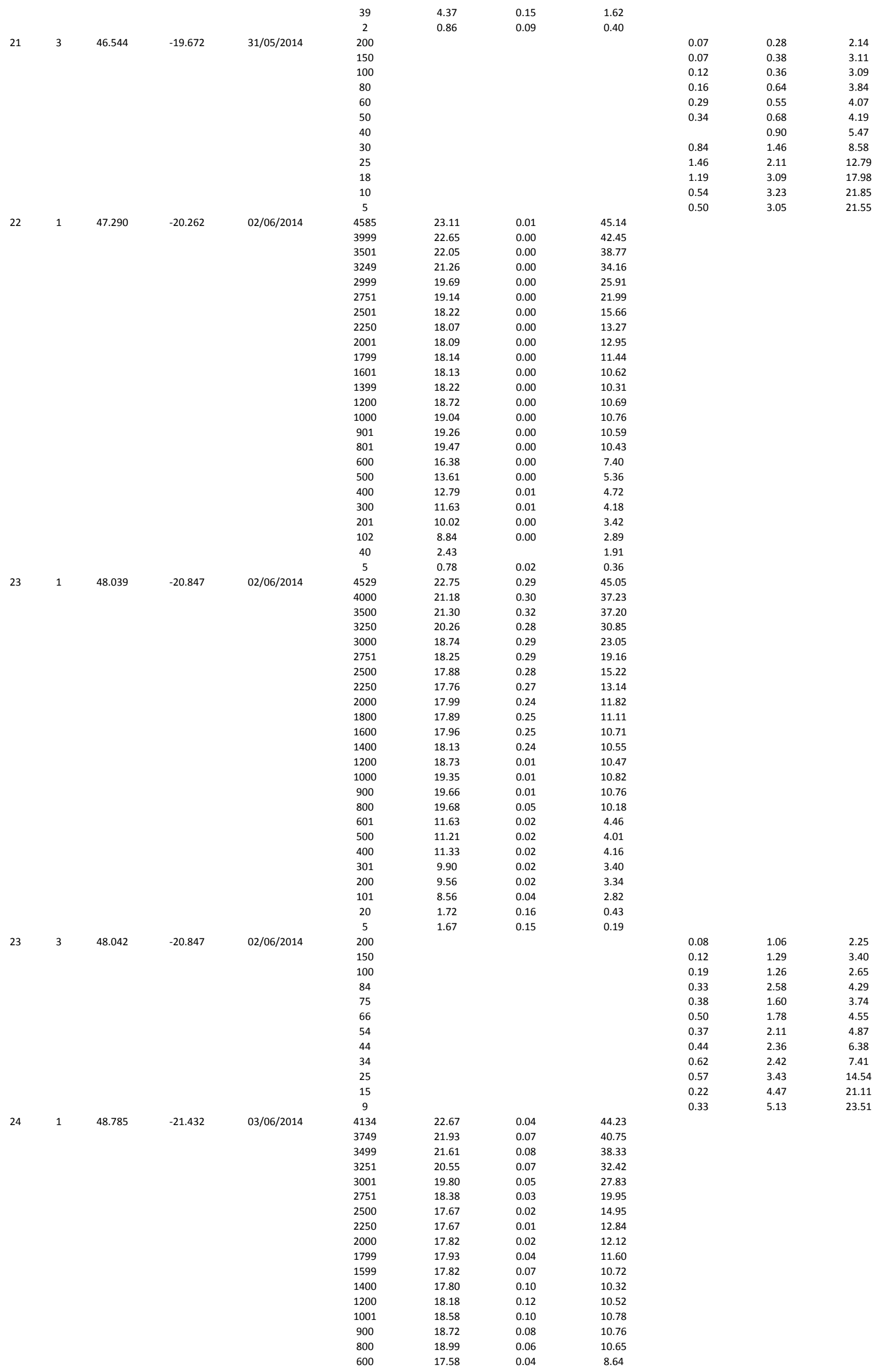




\begin{tabular}{|c|c|c|c|c|c|c|c|}
\hline & & & & & 500 & 13.83 & 0.05 \\
\hline & & & & & 399 & 11.83 & 0.06 \\
\hline & & & & & 300 & 11.57 & 0.05 \\
\hline & & & & & 200 & 11.38 & 0.04 \\
\hline & & & & & 100 & 10.83 & 0.05 \\
\hline & & & & & 40 & 5.02 & 0.21 \\
\hline & & & & & 6 & 3.07 & 0.14 \\
\hline 25 & 1 & 49.529 & -22.017 & 03/06/2014 & 4272 & 22.55 & 0.00 \\
\hline & & & & & 4000 & 22.12 & 0.00 \\
\hline & & & & & 3501 & 20.48 & 0.00 \\
\hline & & & & & 3249 & 19.29 & 0.00 \\
\hline & & & & & 2999 & 18.45 & 0.00 \\
\hline & & & & & 2750 & 18.14 & 0.00 \\
\hline & & & & & 2500 & 17.57 & 0.00 \\
\hline & & & & & 2250 & 17.78 & 0.00 \\
\hline & & & & & 1999 & 17.84 & 0.00 \\
\hline & & & & & 1800 & 17.73 & 0.00 \\
\hline & & & & & 1601 & 17.68 & 0.00 \\
\hline & & & & & 1400 & 17.72 & 0.00 \\
\hline & & & & & 1200 & 18.03 & 0.00 \\
\hline & & & & & 1001 & 18.63 & 0.00 \\
\hline & & & & & 899 & 19.28 & 0.00 \\
\hline & & & & & 799 & 19.76 & 0.00 \\
\hline & & & & & 600 & 16.68 & 0.01 \\
\hline & & & & & 500 & 16.72 & 0.01 \\
\hline & & & & & 400 & 11.85 & 0.02 \\
\hline & & & & & 299 & 10.98 & 0.02 \\
\hline & & & & & 199 & 10.79 & 0.03 \\
\hline & & & & & 100 & 9.39 & 0.04 \\
\hline & & & & & 15 & 3.90 & 0.20 \\
\hline & & & & & 8 & 3.86 & 0.20 \\
\hline 25 & 3 & 49.529 & -22.017 & 03/06/2014 & 200 & & \\
\hline & & & & & 150 & & \\
\hline & & & & & 101 & & \\
\hline & & & & & 80 & & \\
\hline & & & & & 70 & & \\
\hline & & & & & 60 & & \\
\hline & & & & & 50 & & \\
\hline & & & & & 40 & & \\
\hline & & & & & 30 & & \\
\hline & & & & & 20 & & \\
\hline & & & & & 9 & & \\
\hline & & & & & 4 & & \\
\hline 26 & 1 & 50.278 & -22.602 & $04 / 06 / 2014$ & 4192 & 22.33 & 0.00 \\
\hline & & & & & 3500 & 20.44 & 0.00 \\
\hline & & & & & 3251 & 19.69 & 0.00 \\
\hline & & & & & 2999 & 18.48 & 0.00 \\
\hline & & & & & 2749 & 17.65 & 0.00 \\
\hline & & & & & 2499 & 17.42 & 0.00 \\
\hline & & & & & 2300 & 17.40 & 0.00 \\
\hline & & & & & 1998 & 17.62 & 0.00 \\
\hline & & & & & 1800 & 17.68 & 0.00 \\
\hline & & & & & 1600 & 17.70 & 0.00 \\
\hline & & & & & 1400 & 17.63 & 0.00 \\
\hline & & & & & 1200 & 17.66 & 0.00 \\
\hline & & & & & 999 & 17.87 & 0.00 \\
\hline & & & & & 899 & 18.09 & 0.00 \\
\hline & & & & & 750 & 18.40 & 0.01 \\
\hline & & & & & 600 & 19.37 & 0.01 \\
\hline & & & & & 501 & 19.70 & 0.01 \\
\hline & & & & & 400 & 16.57 & 0.02 \\
\hline & & & & & 300 & 13.93 & 0.02 \\
\hline & & & & & 200 & 13.42 & 0.06 \\
\hline & & & & & 150 & 12.88 & \\
\hline & & & & & 100 & 11.49 & \\
\hline & & & & & 35 & 6.33 & 0.55 \\
\hline & & & & & 4 & 6.05 & 0.53 \\
\hline 26 & 7 & 50.278 & -22.605 & 05/06/2014 & 200 & & \\
\hline & & & & & 150 & & \\
\hline & & & & & 100 & & \\
\hline & & & & & 85 & & \\
\hline & & & & & 70 & & \\
\hline & & & & & 60 & & \\
\hline & & & & & 50 & & \\
\hline & & & & & 45 & & \\
\hline & & & & & 35 & & \\
\hline & & & & & 25 & & \\
\hline & & & & & 10 & & \\
\hline & & & & & 7 & & \\
\hline 27 & 1 & 51.030 & -23.191 & 05/06/2014 & 3989 & 22.42 & 0.01 \\
\hline & & & & & 3751 & 22.09 & 0.01 \\
\hline & & & & & 3501 & 21.28 & 0.01 \\
\hline & & & & & 3251 & 19.93 & 0.00 \\
\hline & & & & & 3000 & 18.64 & 0.00 \\
\hline & & & & & 2750 & 17.90 & 0.00 \\
\hline & & & & & 2500 & 17.73 & 0.00 \\
\hline & & & & & 2250 & 17.76 & 0.00 \\
\hline & & & & & 2000 & 17.81 & 0.00 \\
\hline & & & & & 1800 & 17.88 & 0.00 \\
\hline & & & & & 1600 & 17.92 & 0.00 \\
\hline & & & & & 1400 & 17.86 & 0.00 \\
\hline
\end{tabular}




\begin{tabular}{|c|c|c|c|c|c|c|c|}
\hline & & & & & 1199 & 17.81 & 0.01 \\
\hline & & & & & 1000 & 17.86 & 0.01 \\
\hline & & & & & 850 & 18.51 & 0.01 \\
\hline & & & & & 701 & 18.70 & 0.01 \\
\hline & & & & & 600 & 19.05 & 0.01 \\
\hline & & & & & 500 & 19.58 & 0.01 \\
\hline & & & & & 400 & 18.77 & 0.01 \\
\hline & & & & & 301 & 15.18 & 0.02 \\
\hline & & & & & 200 & 14.81 & 0.01 \\
\hline & & & & & 101 & 12.24 & 0.23 \\
\hline & & & & & 40 & 7.29 & 0.16 \\
\hline & & & & & 3 & 5.53 & 0.14 \\
\hline 28 & 1 & 52.025 & -23.973 & $05 / 06 / 2014$ & 3924 & 22.11 & 0.04 \\
\hline & & & & & 3749 & 21.72 & 0.04 \\
\hline & & & & & 3500 & 20.88 & 0.04 \\
\hline & & & & & 3249 & 19.78 & 0.04 \\
\hline & & & & & 3000 & 18.56 & 0.00 \\
\hline & & & & & 2750 & 17.83 & 0.00 \\
\hline & & & & & 2501 & 17.69 & 0.00 \\
\hline & & & & & 2250 & 17.78 & 0.00 \\
\hline & & & & & 1999 & 17.82 & 0.04 \\
\hline & & & & & 1800 & 17.93 & 0.04 \\
\hline & & & & & 1601 & 17.92 & 0.04 \\
\hline & & & & & 1400 & 17.88 & 0.04 \\
\hline & & & & & 1200 & 17.84 & 0.00 \\
\hline & & & & & 1000 & 18.04 & 0.00 \\
\hline & & & & & 850 & 18.43 & 0.00 \\
\hline & & & & & 700 & 18.96 & 0.00 \\
\hline & & & & & 599 & 19.40 & 0.01 \\
\hline & & & & & 501 & 19.56 & 0.01 \\
\hline & & & & & 401 & 15.69 & 0.01 \\
\hline & & & & & 300 & 14.66 & 0.02 \\
\hline & & & & & 200 & 13.06 & 0.02 \\
\hline & & & & & 100 & 9.29 & 0.27 \\
\hline & & & & & 39 & 6.51 & 0.17 \\
\hline & & & & & 6 & 6.13 & 0.16 \\
\hline 29 & 1 & 53.019 & -24.752 & $06 / 06 / 2014$ & 3588 & 20.29 & 0.01 \\
\hline & & & & & 3251 & 18.25 & 0.00 \\
\hline & & & & & 3002 & 17.71 & 0.01 \\
\hline & & & & & 2750 & 17.31 & 0.01 \\
\hline & & & & & 2499 & 17.39 & 0.01 \\
\hline & & & & & 2250 & 17.43 & 0.00 \\
\hline & & & & & 2001 & 17.62 & 0.00 \\
\hline & & & & & 1801 & 17.65 & 0.01 \\
\hline & & & & & 1600 & 17.65 & 0.01 \\
\hline & & & & & 1400 & 17.57 & 0.01 \\
\hline & & & & & 1200 & 17.44 & 0.00 \\
\hline & & & & & 1002 & 17.33 & 0.00 \\
\hline & & & & & 900 & 17.42 & 0.01 \\
\hline & & & & & 801 & 17.57 & 0.00 \\
\hline & & & & & 700 & 17.77 & 0.00 \\
\hline & & & & & 600 & 18.10 & 0.01 \\
\hline & & & & & 501 & 18.66 & 0.01 \\
\hline & & & & & 401 & 17.36 & 0.01 \\
\hline & & & & & 299 & 16.71 & 0.02 \\
\hline & & & & & 200 & 15.86 & 0.13 \\
\hline & & & & & 150 & 15.25 & 0.13 \\
\hline & & & & & 100 & 13.70 & 0.43 \\
\hline & & & & & 25 & 8.06 & 0.17 \\
\hline & & & & & 6 & 6.96 & 0.16 \\
\hline 29 & 3 & 53.020 & -24.752 & $06 / 06 / 2014$ & 201 & & \\
\hline & & & & & 130 & & \\
\hline & & & & & 100 & & \\
\hline & & & & & 80 & & \\
\hline & & & & & 70 & & \\
\hline & & & & & 60 & & \\
\hline & & & & & 50 & & \\
\hline & & & & & 40 & & \\
\hline & & & & & 30 & & \\
\hline & & & & & 20 & & \\
\hline & & & & & 10 & & \\
\hline & & & & & 5 & & \\
\hline 30 & 1 & 54.015 & -25.533 & $06 / 06 / 2014$ & 3106 & 19.40 & 0.00 \\
\hline & & & & & 3106 & 19.40 & 0.01 \\
\hline & & & & & 2750 & 18.10 & 0.01 \\
\hline & & & & & 2501 & 17.16 & 0.01 \\
\hline & & & & & 2247 & 17.22 & 0.00 \\
\hline & & & & & 2002 & 17.45 & 0.00 \\
\hline & & & & & 1801 & 17.59 & 0.00 \\
\hline & & & & & 1599 & 17.77 & 0.04 \\
\hline & & & & & 1399 & 17.62 & 0.04 \\
\hline & & & & & 1203 & 17.36 & 0.04 \\
\hline & & & & & 999 & 17.82 & 0.04 \\
\hline & & & & & 901 & 17.68 & 0.00 \\
\hline & & & & & 801 & 17.88 & 0.00 \\
\hline & & & & & 700 & 17.93 & 0.01 \\
\hline & & & & & 599 & 18.41 & 0.01 \\
\hline & & & & & 500 & 18.82 & 0.01 \\
\hline & & & & & 401 & 18.75 & 0.01 \\
\hline & & & & & 300 & 16.77 & 0.02 \\
\hline & & & & & 199 & 15.31 & 0.02 \\
\hline
\end{tabular}




\begin{tabular}{|c|c|c|c|c|c|c|c|}
\hline & & & & & 149 & 12.43 & 0.32 \\
\hline & & & & & 99 & 11.58 & 0.00 \\
\hline & & & & & 40 & 9.86 & 0.24 \\
\hline & & & & & 17 & 7.58 & 0.17 \\
\hline 31 & 1 & 54.761 & -26.122 & 07/06/2014 & 3661 & 19.35 & 0.01 \\
\hline & & & & & 3501 & 19.19 & 0.00 \\
\hline & & & & & 3239 & 18.08 & 0.00 \\
\hline & & & & & 3000 & 17.67 & 0.00 \\
\hline & & & & & 2752 & 17.33 & 0.00 \\
\hline & & & & & 2500 & 17.31 & 0.01 \\
\hline & & & & & 2250 & 17.52 & 0.01 \\
\hline & & & & & 2002 & 17.54 & 0.00 \\
\hline & & & & & 1801 & 17.76 & 0.00 \\
\hline & & & & & 1598 & 17.75 & 0.00 \\
\hline & & & & & 1399 & 17.71 & 0.00 \\
\hline & & & & & 1202 & 17.58 & 0.00 \\
\hline & & & & & 999 & 17.66 & 0.00 \\
\hline & & & & & 850 & 18.14 & 0.00 \\
\hline & & & & & 701 & 18.47 & 0.01 \\
\hline & & & & & 600 & 18.89 & 0.01 \\
\hline & & & & & 498 & 19.12 & 0.01 \\
\hline & & & & & 400 & 17.88 & 0.01 \\
\hline & & & & & 301 & 14.84 & 0.01 \\
\hline & & & & & 201 & 13.37 & 0.03 \\
\hline & & & & & 152 & 13.65 & 0.03 \\
\hline & & & & & 101 & 13.22 & 0.03 \\
\hline & & & & & 21 & 6.96 & 0.17 \\
\hline & & & & & 16 & 6.70 & 0.17 \\
\hline 32 & 2 & 55.505 & -26.711 & 07/06/2014 & 3273 & 18.50 & 0.00 \\
\hline & & & & & 3000 & 18.22 & 0.00 \\
\hline & & & & & 2799 & 17.74 & 0.00 \\
\hline & & & & & 2500 & 17.46 & 0.00 \\
\hline & & & & & 2250 & 17.54 & 0.00 \\
\hline & & & & & 2000 & 17.59 & 0.00 \\
\hline & & & & & 1700 & 17.73 & 0.00 \\
\hline & & & & & 1549 & 17.73 & 0.00 \\
\hline & & & & & 1399 & 17.00 & 0.00 \\
\hline & & & & & 1200 & 16.86 & 0.00 \\
\hline & & & & & 1000 & 17.08 & 0.00 \\
\hline & & & & & 899 & 17.75 & 0.00 \\
\hline & & & & & 801 & 17.67 & 0.00 \\
\hline & & & & & 700 & 17.52 & 0.00 \\
\hline & & & & & 600 & 17.66 & 0.00 \\
\hline & & & & & 501 & 19.04 & 0.01 \\
\hline & & & & & 450 & 19.11 & 0.01 \\
\hline & & & & & 378 & 16.53 & 0.02 \\
\hline & & & & & 300 & 15.49 & 0.02 \\
\hline & & & & & 201 & 13.87 & 0.03 \\
\hline & & & & & 151 & 13.76 & 0.08 \\
\hline & & & & & 100 & 9.53 & 0.67 \\
\hline & & & & & 31 & 9.17 & 0.27 \\
\hline & & & & & 16 & 7.09 & 0.15 \\
\hline 32 & 15 & 55.515 & -26.704 & 09/06/2014 & 200 & & \\
\hline & & & & & 150 & & \\
\hline & & & & & 100 & & \\
\hline & & & & & 85 & & \\
\hline & & & & & 70 & & \\
\hline & & & & & 55 & & \\
\hline & & & & & 45 & & \\
\hline & & & & & 35 & & \\
\hline & & & & & 25 & & \\
\hline & & & & & 15 & & \\
\hline & & & & & 10 & & \\
\hline & & & & & 4 & & \\
\hline 33 & 1 & 56.252 & -27.292 & 09/06/2014 & 2680 & 18.34 & 0.01 \\
\hline & & & & & 2501 & 17.86 & 0.01 \\
\hline & & & & & 2250 & 17.52 & 0.01 \\
\hline & & & & & 2001 & 17.94 & 0.02 \\
\hline & & & & & 1800 & 18.08 & 0.01 \\
\hline & & & & & 1601 & 18.20 & 0.01 \\
\hline & & & & & 1400 & 18.15 & 0.01 \\
\hline & & & & & 1201 & 18.13 & 0.01 \\
\hline & & & & & 1000 & 18.30 & 0.02 \\
\hline & & & & & 900 & 18.54 & 0.01 \\
\hline & & & & & 801 & 18.84 & 0.01 \\
\hline & & & & & 801 & 18.79 & 0.01 \\
\hline & & & & & 701 & 19.35 & 0.01 \\
\hline & & & & & 601 & 17.53 & 0.02 \\
\hline & & & & & 501 & 15.62 & 0.01 \\
\hline & & & & & 401 & 14.78 & 0.02 \\
\hline & & & & & 300 & 13.42 & 0.03 \\
\hline & & & & & 201 & 12.94 & 0.06 \\
\hline & & & & & 152 & 13.19 & 0.08 \\
\hline & & & & & 101 & 10.99 & 0.35 \\
\hline & & & & & 50 & 8.38 & 0.64 \\
\hline & & & & & 25 & 5.76 & 0.15 \\
\hline & & & & & 5 & 5.63 & 0.15 \\
\hline 34 & 1 & 57.004 & -27.878 & 09/06/2014 & 2784 & 16.89 & 0.00 \\
\hline & & & & & 2700 & 17.06 & 0.00 \\
\hline & & & & & 2500 & 17.17 & 0.00 \\
\hline & & & & & 2250 & 17.15 & 0.00 \\
\hline
\end{tabular}




\begin{tabular}{|c|c|c|c|c|c|c|}
\hline & & & & & & \\
\hline & & & & & 2000 & 17.40 \\
\hline & & & & & 1800 & 17.62 \\
\hline & & & & & 1599 & 17.87 \\
\hline & & & & & 1400 & 17.85 \\
\hline & & & & & 1200 & 17.81 \\
\hline & & & & & 1000 & 17.94 \\
\hline & & & & & 850 & 18.23 \\
\hline & & & & & 700 & 18.64 \\
\hline & & & & & 601 & 18.88 \\
\hline & & & & & 449 & 18.97 \\
\hline & & & & & 381 & 17.76 \\
\hline & & & & & 300 & 15.30 \\
\hline & & & & & 200 & 15.47 \\
\hline & & & & & 150 & 14.18 \\
\hline & & & & & 100 & 13.25 \\
\hline & & & & & 27 & 7.32 \\
\hline & & & & & 5 & 6.96 \\
\hline 34 & 3 & 57.004 & -27.879 & 09/06/2014 & 199 & \\
\hline & & & & & 150 & \\
\hline & & & & & 100 & \\
\hline & & & & & 80 & \\
\hline & & & & & 69 & \\
\hline & & & & & 60 & \\
\hline & & & & & 50 & \\
\hline & & & & & 40 & \\
\hline & & & & & 30 & \\
\hline & & & & & 20 & \\
\hline & & & & & 10 & \\
\hline & & & & & 4 & \\
\hline 35 & 1 & 57.675 & -28.730 & $10 / 06 / 2014$ & 2496 & 16.44 \\
\hline & & & & & 2300 & 16.43 \\
\hline & & & & & 2101 & 17.15 \\
\hline & & & & & 2000 & 17.22 \\
\hline & & & & & 1800 & 17.40 \\
\hline & & & & & 1600 & 17.67 \\
\hline & & & & & 1500 & 17.78 \\
\hline & & & & & 1401 & 17.79 \\
\hline & & & & & 1300 & 17.70 \\
\hline & & & & & 1200 & 17.67 \\
\hline & & & & & 1100 & 17.66 \\
\hline & & & & & 1001 & 17.73 \\
\hline & & & & & 899 & 17.79 \\
\hline & & & & & 800 & 18.04 \\
\hline & & & & & 700 & 18.44 \\
\hline & & & & & 600 & 18.79 \\
\hline & & & & & 500 & 18.56 \\
\hline & & & & & 400 & 16.73 \\
\hline & & & & & 301 & 14.99 \\
\hline & & & & & 220 & 14.71 \\
\hline & & & & & 149 & 14.81 \\
\hline & & & & & 100 & 14.81 \\
\hline & & & & & 39 & 10.49 \\
\hline & & & & & 5 & 7.25 \\
\hline 36 & 1 & 58.207 & -29.725 & $10 / 06 / 2014$ & 2244 & 16.22 \\
\hline & & & & & 2244 & \\
\hline & & & & & 2101 & 16.26 \\
\hline & & & & & 2100 & 16.30 \\
\hline & & & & & 2000 & 16.39 \\
\hline & & & & & 1801 & 16.84 \\
\hline & & & & & 1600 & 17.18 \\
\hline & & & & & 1400 & 17.46 \\
\hline & & & & & 1300 & 17.61 \\
\hline & & & & & 1200 & 17.63 \\
\hline & & & & & 1101 & 17.65 \\
\hline & & & & & 1001 & 17.55 \\
\hline & & & & & 901 & 17.72 \\
\hline & & & & & 800 & 17.84 \\
\hline & & & & & 700 & 18.25 \\
\hline & & & & & 600 & 18.42 \\
\hline & & & & & 500 & 18.55 \\
\hline & & & & & 401 & 17.04 \\
\hline & & & & & 301 & 14.87 \\
\hline & & & & & 200 & 15.01 \\
\hline & & & & & 150 & 14.83 \\
\hline & & & & & 100 & 14.37 \\
\hline & & & & & 20 & 7.89 \\
\hline & & & & & 5 & 7.46 \\
\hline 37 & 1 & 58.406 & -30.095 & $10 / 06 / 2014$ & 1597 & 16.75 \\
\hline & & & & & 1500 & 16.83 \\
\hline & & & & & 1400 & 16.90 \\
\hline & & & & & 1300 & 16.92 \\
\hline & & & & & 1200 & 17.05 \\
\hline & & & & & 1101 & 17.39 \\
\hline & & & & & 1001 & 17.54 \\
\hline & & & & & 900 & 17.59 \\
\hline & & & & & 800 & 17.89 \\
\hline & & & & & 700 & 18.30 \\
\hline & & & & & 600 & 18.46 \\
\hline & & & & & 499 & 17.75 \\
\hline & & & & & 400 & 16.56 \\
\hline & & & & & 301 & 14.91 \\
\hline
\end{tabular}




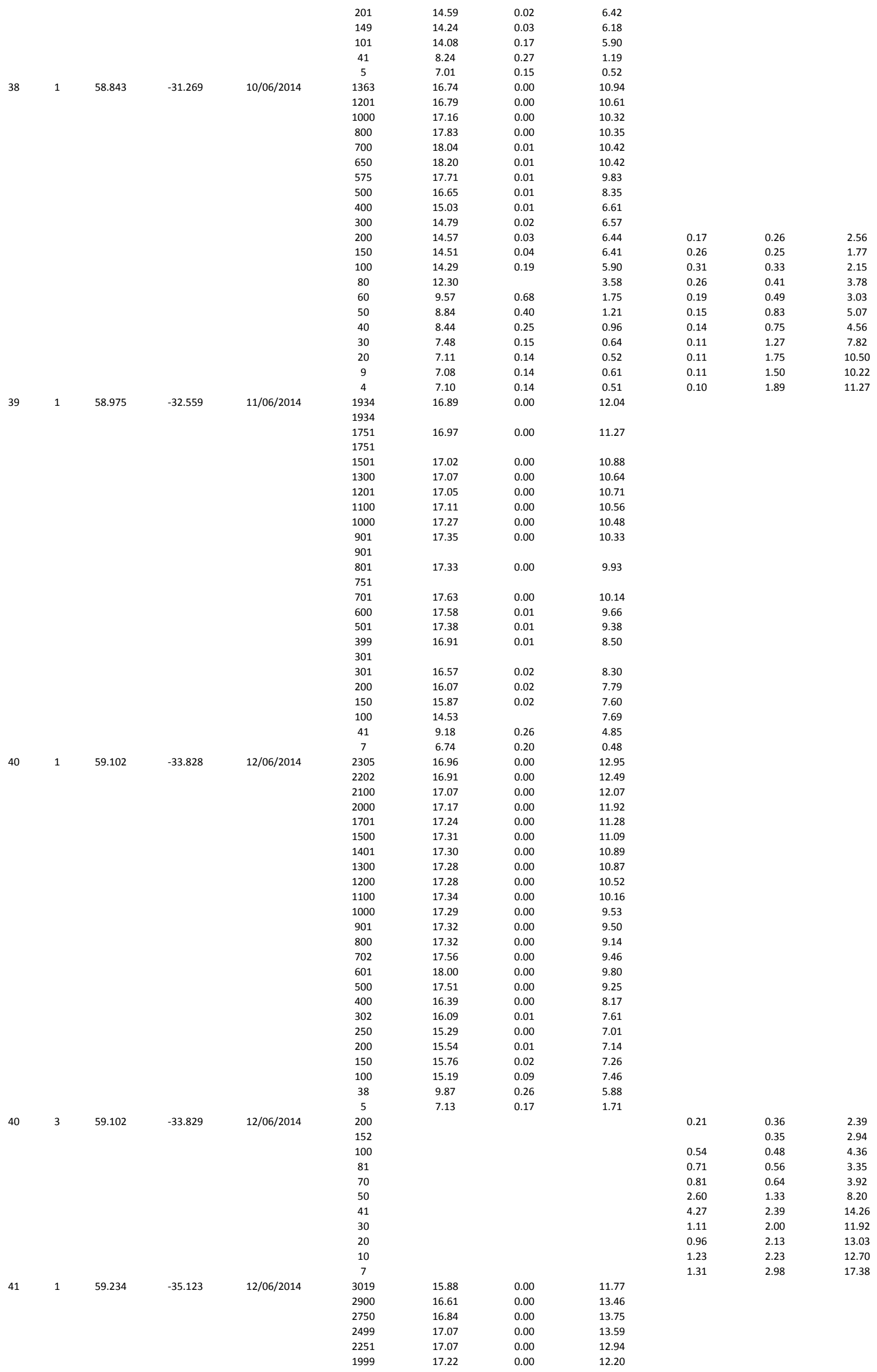




\begin{tabular}{|c|c|c|c|c|c|c|}
\hline & & & & & 1800 & 17.18 \\
\hline & & & & & 1599 & 17.15 \\
\hline & & & & & 1401 & 17.47 \\
\hline & & & & & 1200 & 17.47 \\
\hline & & & & & 1001 & 17.57 \\
\hline & & & & & 900 & 17.41 \\
\hline & & & & & 800 & 17.52 \\
\hline & & & & & 700 & 17.53 \\
\hline & & & & & 599 & 17.56 \\
\hline & & & & & 499 & 17.79 \\
\hline & & & & & 401 & 17.65 \\
\hline & & & & & 300 & 17.06 \\
\hline & & & & & 201 & 16.51 \\
\hline & & & & & 150 & 16.26 \\
\hline & & & & & 100 & 15.80 \\
\hline & & & & & 40 & 10.77 \\
\hline & & & & & 5 & 7.45 \\
\hline 42 & 1 & 59.363 & -36.399 & $12 / 06 / 2014$ & 3138 & 14.23 \\
\hline & & & & & 3101 & 15.02 \\
\hline & & & & & 2899 & 16.50 \\
\hline & & & & & 2501 & 16.90 \\
\hline & & & & & 2248 & 16.99 \\
\hline & & & & & 2000 & 17.04 \\
\hline & & & & & 1800 & 17.15 \\
\hline & & & & & 1600 & 17.21 \\
\hline & & & & & 1400 & 17.20 \\
\hline & & & & & 1200 & 17.01 \\
\hline & & & & & 1049 & 16.55 \\
\hline & & & & & 900 & 16.40 \\
\hline & & & & & 800 & 16.25 \\
\hline & & & & & 700 & 16.20 \\
\hline & & & & & 599 & 16.40 \\
\hline & & & & & 500 & 16.35 \\
\hline & & & & & 401 & 16.20 \\
\hline & & & & & 301 & 17.01 \\
\hline & & & & & 251 & 16.63 \\
\hline & & & & & 200 & 16.71 \\
\hline & & & & & 151 & 16.52 \\
\hline & & & & & 101 & 15.69 \\
\hline & & & & & 28 & 8.51 \\
\hline & & & & & 7 & 8.11 \\
\hline 42 & 3 & 59.363 & -36.399 & $12 / 06 / 2014$ & 200 & \\
\hline & & & & & 150 & \\
\hline & & & & & 100 & \\
\hline & & & & & 80 & \\
\hline & & & & & 70 & \\
\hline & & & & & 60 & \\
\hline & & & & & 50 & \\
\hline & & & & & 40 & \\
\hline & & & & & 30 & \\
\hline & & & & & 22 & \\
\hline & & & & & 10 & \\
\hline & & & & & 5 & \\
\hline 43 & 1 & 59.491 & -37.681 & $13 / 06 / 2014$ & 3150 & 14.26 \\
\hline & & & & & 3000 & 15.86 \\
\hline & & & & & 2750 & 17.08 \\
\hline & & & & & 2500 & 17.14 \\
\hline & & & & & 2251 & 17.11 \\
\hline & & & & & 2001 & 17.31 \\
\hline & & & & & 1800 & 17.37 \\
\hline & & & & & 1600 & 17.25 \\
\hline & & & & & 1400 & 17.47 \\
\hline & & & & & 1200 & 16.93 \\
\hline & & & & & 1000 & 16.74 \\
\hline & & & & & 900 & 16.41 \\
\hline & & & & & 802 & 16.36 \\
\hline & & & & & 700 & 16.65 \\
\hline & & & & & 600 & 16.82 \\
\hline & & & & & 500 & 16.89 \\
\hline & & & & & 400 & 16.65 \\
\hline & & & & & 300 & 16.86 \\
\hline & & & & & 201 & 16.03 \\
\hline & & & & & 150 & 15.94 \\
\hline & & & & & 101 & 15.91 \\
\hline & & & & & 39 & 11.49 \\
\hline & & & & & 5 & 8.08 \\
\hline & & & & & 5 & \\
\hline 44 & 1 & 59.623 & -38.954 & $13 / 06 / 2014$ & 2966 & 9.54 \\
\hline & & & & & 2900 & 13.82 \\
\hline & & & & & 2800 & 13.78 \\
\hline & & & & & 2601 & 14.13 \\
\hline & & & & & 2501 & 16.83 \\
\hline & & & & & 2249 & 16.89 \\
\hline & & & & & 2000 & 16.98 \\
\hline & & & & & 1800 & 17.01 \\
\hline & & & & & 1600 & \\
\hline & & & & & 1401 & 17.25 \\
\hline & & & & & 1101 & 17.25 \\
\hline & & & & & 1001 & 17.01 \\
\hline & & & & & 900 & 17.09 \\
\hline & & & & & 800 & 16.45 \\
\hline
\end{tabular}




\begin{tabular}{|c|c|c|c|c|c|c|c|}
\hline & & & & & 699 & 16.39 & 0.01 \\
\hline & & & & & 600 & 16.40 & 0.01 \\
\hline & & & & & 499 & 16.27 & 0.00 \\
\hline & & & & & 399 & 16.41 & 0.01 \\
\hline & & & & & 301 & 16.30 & 0.01 \\
\hline & & & & & 201 & 16.47 & 0.02 \\
\hline & & & & & 150 & 16.40 & 0.03 \\
\hline & & & & & 99 & 16.46 & 0.04 \\
\hline & & & & & 26 & 16.47 & 0.05 \\
\hline & & & & & 10 & 10.10 & 0.11 \\
\hline 44 & 3 & 59.623 & -38.954 & $13 / 06 / 2014$ & 200 & & \\
\hline & & & & & 150 & & \\
\hline & & & & & 100 & & \\
\hline & & & & & 80 & & \\
\hline & & & & & 60 & & \\
\hline & & & & & 50 & & \\
\hline & & & & & 40 & & \\
\hline & & & & & 30 & & \\
\hline & & & & & 20 & & \\
\hline & & & & & 15 & & \\
\hline & & & & & 10 & & \\
\hline & & & & & 5 & & \\
\hline 45 & 1 & 59.491 & -37.682 & $15 / 06 / 2014$ & 3152 & 14.20 & 0.01 \\
\hline & & & & & 3000 & 15.97 & 0.00 \\
\hline & & & & & 2753 & 16.65 & 0.00 \\
\hline & & & & & 2500 & 17.06 & 0.00 \\
\hline & & & & & 2251 & 17.07 & 0.00 \\
\hline & & & & & 2001 & 17.22 & 0.00 \\
\hline & & & & & 1800 & 17.12 & 0.01 \\
\hline & & & & & 1599 & 17.27 & 0.01 \\
\hline & & & & & 1400 & 17.37 & 0.00 \\
\hline & & & & & 1200 & 16.54 & 0.02 \\
\hline & & & & & 1000 & 16.42 & 0.01 \\
\hline & & & & & 901 & 16.40 & 0.01 \\
\hline & & & & & 799 & 16.61 & 0.01 \\
\hline & & & & & 700 & 16.27 & 0.02 \\
\hline & & & & & 600 & 16.47 & 0.01 \\
\hline & & & & & 499 & 16.95 & 0.01 \\
\hline & & & & & 399 & 16.67 & 0.01 \\
\hline & & & & & 299 & 16.74 & 0.01 \\
\hline & & & & & 200 & 15.87 & 0.02 \\
\hline & & & & & 148 & 15.77 & 0.04 \\
\hline & & & & & 100 & 15.71 & 0.04 \\
\hline & & & & & 41 & 14.07 & 0.28 \\
\hline & & & & & 5 & 8.17 & 0.25 \\
\hline & & & & & 5 & & \\
\hline 46 & 1 & 59.624 & -38.958 & $15 / 06 / 2014$ & 2966 & 13.91 & 0.01 \\
\hline & & & & & 2751 & 14.48 & 0.01 \\
\hline & & & & & 2501 & 16.68 & 0.01 \\
\hline & & & & & 2252 & 16.88 & 0.01 \\
\hline & & & & & 2000 & 16.98 & 0.00 \\
\hline & & & & & 1801 & 17.06 & 0.00 \\
\hline & & & & & 1601 & 17.27 & 0.01 \\
\hline & & & & & 1401 & 17.30 & 0.00 \\
\hline & & & & & 1202 & 17.14 & 0.00 \\
\hline & & & & & 1001 & 16.50 & 0.00 \\
\hline & & & & & 850 & 16.24 & 0.01 \\
\hline & & & & & 701 & 16.06 & 0.01 \\
\hline & & & & & 601 & 16.29 & 0.01 \\
\hline & & & & & 499 & 16.38 & 0.01 \\
\hline & & & & & 400 & 16.26 & 0.01 \\
\hline & & & & & 300 & 16.25 & 0.01 \\
\hline & & & & & 199 & 16.29 & 0.02 \\
\hline & & & & & 150 & 16.42 & 0.04 \\
\hline & & & & & 100 & 16.28 & 0.06 \\
\hline & & & & & 80 & 16.37 & 0.12 \\
\hline & & & & & 60 & 16.10 & \\
\hline & & & & & 40 & 13.31 & 0.18 \\
\hline & & & & & 20 & 9.30 & 0.12 \\
\hline & & & & & 5 & 8.81 & 0.12 \\
\hline 47 & 1 & 59.722 & -40.253 & $15 / 06 / 2014$ & 2687 & 13.87 & 0.00 \\
\hline & & & & & 2685 & 13.93 & 0.01 \\
\hline & & & & & 2599 & 13.98 & 0.00 \\
\hline & & & & & 2501 & 15.58 & 0.00 \\
\hline & & & & & 2250 & 16.81 & 0.00 \\
\hline & & & & & 2000 & 16.94 & 0.00 \\
\hline & & & & & 1800 & 16.94 & 0.00 \\
\hline & & & & & 1601 & 17.02 & 0.00 \\
\hline & & & & & 1401 & 17.05 & 0.00 \\
\hline & & & & & 1200 & 17.22 & 0.00 \\
\hline & & & & & 1001 & 16.94 & 0.00 \\
\hline & & & & & 901 & 16.53 & 0.00 \\
\hline & & & & & 800 & 16.26 & 0.00 \\
\hline & & & & & 701 & 16.56 & 0.00 \\
\hline & & & & & 600 & 16.38 & 0.00 \\
\hline & & & & & 501 & 16.33 & 0.01 \\
\hline & & & & & 399 & 16.07 & 0.00 \\
\hline & & & & & 300 & 16.24 & 0.01 \\
\hline & & & & & 201 & 16.28 & 0.03 \\
\hline & & & & & 150 & 16.13 & 0.09 \\
\hline & & & & & 101 & 16.33 & 0.04 \\
\hline
\end{tabular}




\begin{tabular}{|c|c|c|c|c|c|c|c|}
\hline & & & & & 38 & 10.69 & 0.18 \\
\hline & & & & & 5 & 8.85 & 0.11 \\
\hline & & & & & 5 & & \\
\hline & & & & & 2295 & 14.29 & 0.01 \\
\hline 48 & 1 & 59.758 & -40.905 & $15 / 06 / 2014$ & 2200 & 14.42 & 0.01 \\
\hline & & & & & 2100 & 15.11 & 0.00 \\
\hline & & & & & 2001 & 15.95 & 0.00 \\
\hline & & & & & 1800 & 16.11 & 0.00 \\
\hline & & & & & 1601 & 16.14 & 0.00 \\
\hline & & & & & 1501 & 16.37 & 0.00 \\
\hline & & & & & 1401 & 16.62 & 0.00 \\
\hline & & & & & 1301 & 16.64 & 0.00 \\
\hline & & & & & 1200 & 16.68 & 0.00 \\
\hline & & & & & 1100 & 16.90 & 0.00 \\
\hline & & & & & 1000 & 16.72 & 0.00 \\
\hline & & & & & 901 & 16.77 & 0.00 \\
\hline & & & & & 802 & 16.91 & 0.00 \\
\hline & & & & & 700 & 16.87 & 0.00 \\
\hline & & & & & 601 & 16.72 & 0.01 \\
\hline & & & & & 548 & 16.51 & 0.00 \\
\hline & & & & & 400 & 16.42 & 0.01 \\
\hline & & & & & 301 & 16.14 & 0.01 \\
\hline & & & & & 201 & 15.55 & 0.07 \\
\hline & & & & & 150 & 15.15 & 0.21 \\
\hline & & & & & 101 & 14.47 & \\
\hline & & & & & 40 & 10.79 & 0.18 \\
\hline & & & & & 6 & 9.53 & 0.11 \\
\hline 49 & 1 & 59.773 & -41.297 & $15 / 06 / 2014$ & 2055 & 14.93 & 0.00 \\
\hline & & & & & 2001 & 14.94 & 0.01 \\
\hline & & & & & 1801 & 16.29 & 0.00 \\
\hline & & & & & 1700 & 15.82 & 0.01 \\
\hline & & & & & 1601 & 16.19 & 0.00 \\
\hline & & & & & 1500 & 16.28 & 0.00 \\
\hline & & & & & 1403 & 16.37 & 0.01 \\
\hline & & & & & 1301 & 16.71 & 0.01 \\
\hline & & & & & 1202 & & \\
\hline & & & & & 1101 & 16.60 & 0.01 \\
\hline & & & & & 1002 & 16.61 & 0.01 \\
\hline & & & & & 901 & 16.33 & 0.01 \\
\hline & & & & & 801 & 16.58 & 0.01 \\
\hline & & & & & 701 & 16.66 & 0.00 \\
\hline & & & & & 601 & 16.55 & 0.00 \\
\hline & & & & & 500 & 16.32 & 0.01 \\
\hline & & & & & 400 & 16.10 & 0.01 \\
\hline & & & & & 301 & 16.04 & 0.02 \\
\hline & & & & & 251 & 15.62 & 0.04 \\
\hline & & & & & 201 & 15.63 & 0.09 \\
\hline & & & & & 150 & 14.51 & 0.64 \\
\hline & & & & & 86 & 13.71 & \\
\hline & & & & & 40 & 9.66 & 0.14 \\
\hline & & & & & 6 & 9.40 & 0.12 \\
\hline 49 & 3 & 59.773 & -41.297 & $15 / 06 / 2014$ & 200 & & \\
\hline & & & & & 150 & & \\
\hline & & & & & 100 & & \\
\hline & & & & & 80 & & \\
\hline & & & & & 70 & & \\
\hline & & & & & 60 & & \\
\hline & & & & & 50 & & \\
\hline & & & & & 40 & & \\
\hline & & & & & 30 & & \\
\hline & & & & & 22 & & \\
\hline & & & & & 10 & & \\
\hline & & & & & 5 & & \\
\hline 50 & 1 & 59.795 & -41.730 & $16 / 06 / 2014$ & 1859 & 14.73 & 0.02 \\
\hline & & & & & 1801 & 15.27 & 0.02 \\
\hline & & & & & 1801 & 15.46 & 0.01 \\
\hline & & & & & 1700 & 15.67 & 0.00 \\
\hline & & & & & 1500 & 16.06 & 0.01 \\
\hline & & & & & 1400 & 16.34 & 0.01 \\
\hline & & & & & 1300 & 16.00 & 0.01 \\
\hline & & & & & 1200 & 16.31 & 0.01 \\
\hline & & & & & 1100 & & \\
\hline & & & & & 1000 & 16.40 & 0.01 \\
\hline & & & & & 902 & 16.30 & 0.00 \\
\hline & & & & & 800 & 16.29 & 0.01 \\
\hline & & & & & 700 & 16.27 & 0.01 \\
\hline & & & & & 600 & 16.25 & 0.01 \\
\hline & & & & & 501 & 16.27 & 0.00 \\
\hline & & & & & 400 & 15.99 & 0.02 \\
\hline & & & & & 300 & 15.73 & 0.02 \\
\hline & & & & & 201 & 15.52 & 0.02 \\
\hline & & & & & 151 & 15.10 & 0.17 \\
\hline & & & & & 100 & 14.11 & \\
\hline & & & & & 40 & 9.29 & 0.59 \\
\hline & & & & & 6 & 9.74 & 0.44 \\
\hline 51 & 1 & 59.799 & -42.003 & $16 / 06 / 2014$ & 1732 & 15.53 & 0.00 \\
\hline & & & & & 1702 & 15.38 & 0.01 \\
\hline & & & & & 1600 & 15.58 & 0.00 \\
\hline & & & & & 1500 & 15.60 & 0.01 \\
\hline & & & & & 1400 & 16.06 & 0.00 \\
\hline & & & & & 1299 & 16.11 & 0.01 \\
\hline
\end{tabular}




\begin{tabular}{|c|c|c|c|c|c|c|c|}
\hline & & & & & 1200 & 16.28 & 0.00 \\
\hline & & & & & 1101 & 16.31 & 0.00 \\
\hline & & & & & 1001 & 16.32 & 0.00 \\
\hline & & & & & 900 & 16.33 & 0.01 \\
\hline & & & & & 751 & 16.06 & 0.02 \\
\hline & & & & & 600 & 16.08 & 0.01 \\
\hline & & & & & 500 & 16.09 & 0.02 \\
\hline & & & & & 399 & 15.75 & 0.02 \\
\hline & & & & & 301 & 15.48 & 0.02 \\
\hline & & & & & 202 & 15.21 & 0.06 \\
\hline & & & & & 151 & 14.72 & 0.60 \\
\hline & & & & & 102 & 13.68 & 0.30 \\
\hline & & & & & 40 & 9.28 & 0.16 \\
\hline & & & & & 6 & 9.90 & 0.16 \\
\hline 52 & 1 & 59.808 & -42.238 & $16 / 06 / 2014$ & 1200 & 15.96 & 0.01 \\
\hline & & & & & 1200 & 16.06 & 0.01 \\
\hline & & & & & 1101 & 16.11 & 0.01 \\
\hline & & & & & 1000 & 16.11 & 0.00 \\
\hline & & & & & 900 & 15.89 & 0.00 \\
\hline & & & & & 798 & 15.61 & 0.02 \\
\hline & & & & & 700 & 15.31 & 0.05 \\
\hline & & & & & 600 & 14.57 & 0.28 \\
\hline & & & & & 500 & 14.60 & \\
\hline & & & & & 401 & 14.59 & \\
\hline & & & & & 300 & 13.98 & \\
\hline & & & & & 199 & 13.45 & \\
\hline & & & & & 149 & 12.71 & \\
\hline & & & & & 100 & 12.61 & \\
\hline & & & & & 74 & 12.47 & \\
\hline & & & & & 49 & 12.10 & \\
\hline & & & & & 25 & 10.16 & 0.61 \\
\hline & & & & & 11 & 9.74 & 0.54 \\
\hline & & & & & 5 & 9.40 & 0.34 \\
\hline 53 & 1 & 59.902 & -43.015 & $16 / 06 / 2014$ & 159 & 7.14 & 0.10 \\
\hline & & & & & 100 & 6.53 & 0.08 \\
\hline & & & & & 74 & & \\
\hline & & & & & 50 & 3.67 & 0.04 \\
\hline & & & & & 29 & & \\
\hline & & & & & 25 & & \\
\hline & & & & & 10 & & \\
\hline & & & & & 4 & & \\
\hline 54 & 1 & 59.871 & -42.814 & $17 / 06 / 2014$ & 178 & 11.63 & 0.64 \\
\hline & & & & & 99 & 8.93 & 0.19 \\
\hline & & & & & 50 & 5.77 & 0.11 \\
\hline & & & & & 5 & 2.48 & 0.07 \\
\hline 55 & 1 & 59.831 & -42.520 & $17 / 06 / 2014$ & 220 & 12.05 & 0.53 \\
\hline & & & & & 150 & 10.44 & 0.59 \\
\hline & & & & & 100 & 9.26 & 0.43 \\
\hline & & & & & 50 & 9.41 & 0.26 \\
\hline & & & & & 6 & 8.16 & 0.17 \\
\hline 56 & 1 & 59.823 & -42.399 & $17 / 06 / 2014$ & 298 & 12.55 & -0.25 \\
\hline & & & & & 200 & 11.67 & -0.22 \\
\hline & & & & & 150 & 9.96 & 0.53 \\
\hline & & & & & 100 & 8.58 & 0.60 \\
\hline & & & & & 81 & 8.51 & 0.51 \\
\hline & & & & & 70 & & \\
\hline & & & & & 61 & 9.14 & 0.33 \\
\hline & & & & & 50 & & \\
\hline & & & & & 40 & 8.85 & 0.32 \\
\hline & & & & & 31 & & \\
\hline & & & & & 20 & 8.50 & 0.28 \\
\hline & & & & & 9 & 7.64 & 0.17 \\
\hline & & & & & 6 & & \\
\hline 57 & 1 & 59.818 & -42.313 & $17 / 06 / 2014$ & 559 & 13.40 & 0.64 \\
\hline & & & & & 401 & 13.18 & 0.62 \\
\hline & & & & & 200 & 11.62 & 0.63 \\
\hline & & & & & 101 & 9.89 & 0.41 \\
\hline & & & & & 51 & 9.30 & 0.22 \\
\hline & & & & & 4 & 6.63 & 0.12 \\
\hline 58 & 1 & 59.816 & -42.276 & $17 / 06 / 2014$ & 879 & 16.14 & 0.01 \\
\hline & & & & & 850 & 16.04 & 0.01 \\
\hline & & & & & 798 & 15.94 & 0.01 \\
\hline & & & & & 700 & 15.77 & 0.02 \\
\hline & & & & & 601 & 15.41 & 0.07 \\
\hline & & & & & 498 & 14.29 & 0.26 \\
\hline & & & & & 401 & 13.18 & 0.64 \\
\hline & & & & & 300 & 12.31 & 0.64 \\
\hline & & & & & 200 & 11.56 & 0.63 \\
\hline & & & & & 100 & 10.37 & 0.60 \\
\hline & & & & & 50 & 8.97 & 0.25 \\
\hline & & & & & 6 & 8.99 & 0.25 \\
\hline 60 & 3 & 59.799 & -42.014 & $17 / 06 / 2014$ & 1734 & 14.87 & 0.00 \\
\hline & & & & & 1400 & 15.24 & 0.00 \\
\hline & & & & & 1202 & 15.66 & 0.00 \\
\hline & & & & & 1000 & 15.85 & 0.00 \\
\hline & & & & & 800 & 15.60 & 0.00 \\
\hline & & & & & 500 & 15.54 & 0.01 \\
\hline & & & & & 252 & 15.01 & 0.01 \\
\hline & & & & & 152 & 14.45 & 0.13 \\
\hline & & & & & 100 & 13.52 & 0.27 \\
\hline & & & & & 51 & 10.78 & 0.20 \\
\hline
\end{tabular}




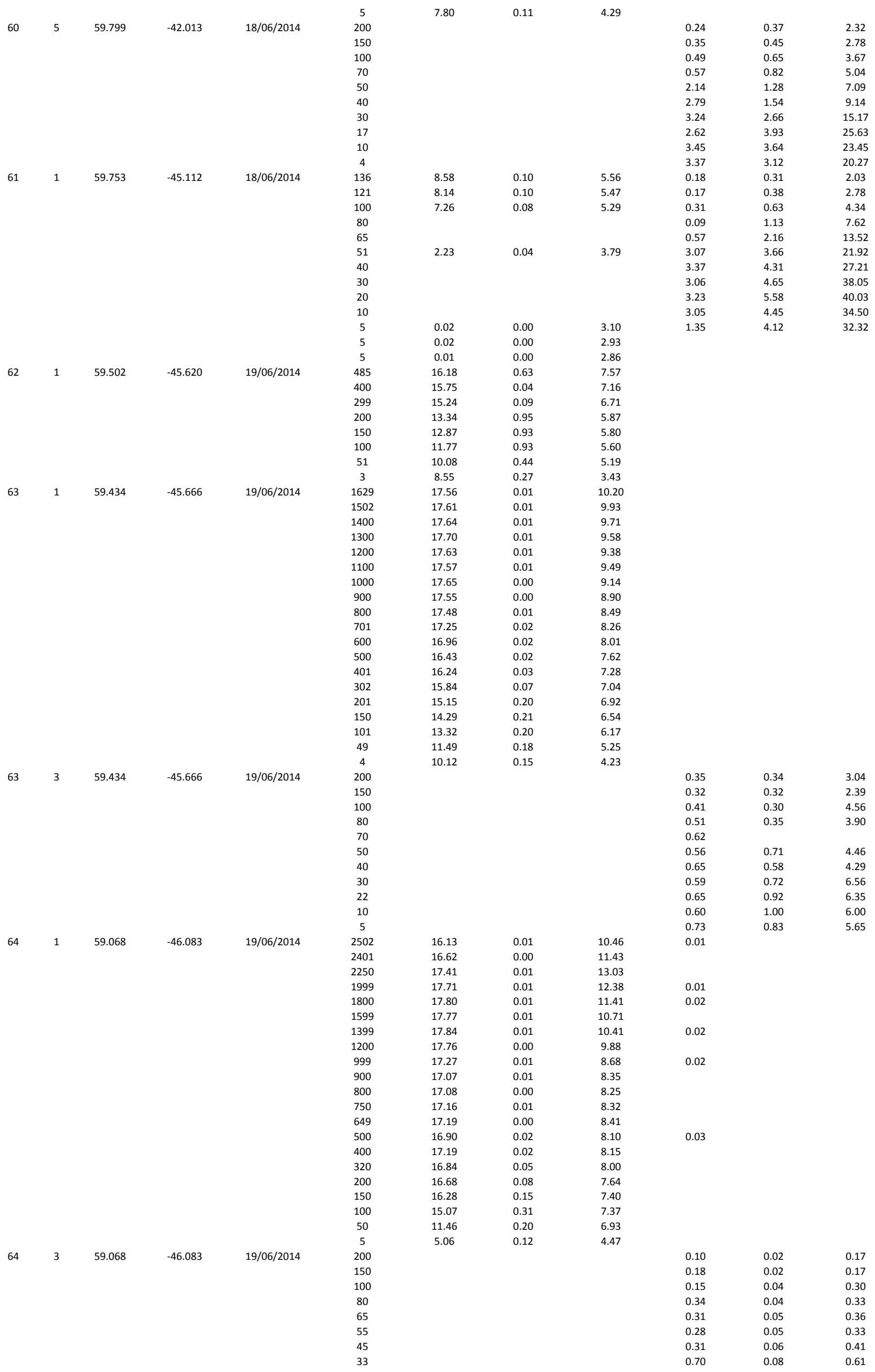




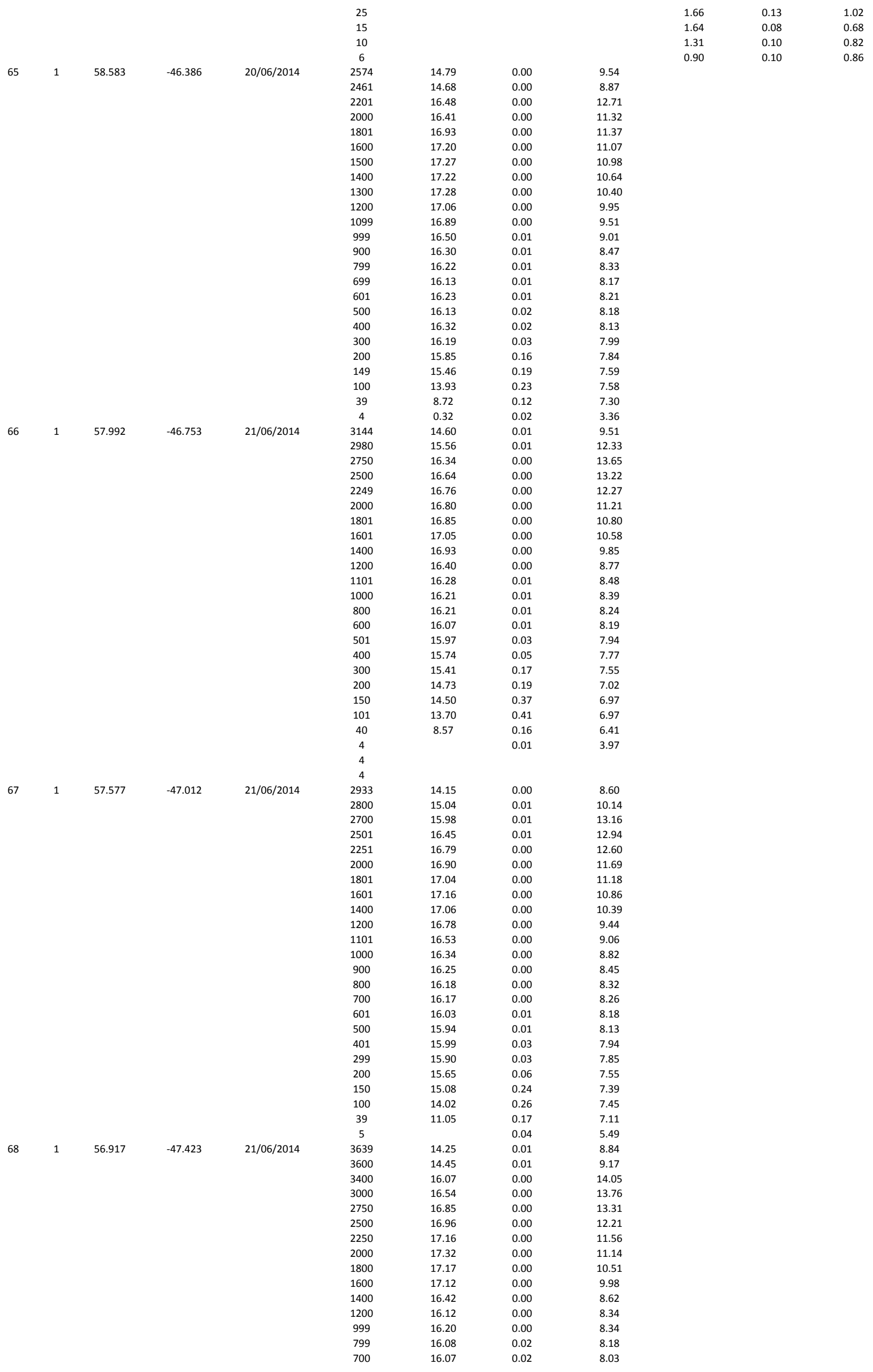




\begin{tabular}{|c|c|c|c|c|c|c|c|}
\hline & & & & & 600 & 16.04 & 0.01 \\
\hline & & & & & 500 & 15.96 & 0.03 \\
\hline & & & & & 400 & 16.11 & 0.03 \\
\hline & & & & & 300 & 15.89 & 0.02 \\
\hline & & & & & 200 & 15.70 & 0.05 \\
\hline & & & & & 150 & 15.48 & 0.09 \\
\hline & & & & & 100 & 15.20 & 0.40 \\
\hline & & & & & 50 & 9.51 & 0.21 \\
\hline & & & & & 4 & & 0.00 \\
\hline 68 & 3 & 56.913 & -47.419 & $21 / 06 / 2014$ & 200 & & \\
\hline & & & & & 150 & & \\
\hline & & & & & 100 & & \\
\hline & & & & & 80 & & \\
\hline & & & & & 70 & & \\
\hline & & & & & 60 & & \\
\hline & & & & & 50 & & \\
\hline & & & & & 40 & & \\
\hline & & & & & 30 & & \\
\hline & & & & & 20 & & \\
\hline & & & & & 10 & & \\
\hline & & & & & 3 & & \\
\hline 69 & 1 & 55.842 & -48.093 & $22 / 06 / 2014$ & 3745 & 14.37 & 0.00 \\
\hline & & & & & 3701 & 14.34 & 0.01 \\
\hline & & & & & 3500 & 15.40 & 0.00 \\
\hline & & & & & 3249 & 16.39 & 0.00 \\
\hline & & & & & 2999 & 16.62 & 0.00 \\
\hline & & & & & 2725 & 16.82 & 0.00 \\
\hline & & & & & 2500 & 16.87 & 0.00 \\
\hline & & & & & 2250 & 17.17 & 0.00 \\
\hline & & & & & 2000 & 17.10 & 0.00 \\
\hline & & & & & 1800 & 17.32 & 0.00 \\
\hline & & & & & 1600 & 16.78 & 0.00 \\
\hline & & & & & 1400 & 16.14 & 0.01 \\
\hline & & & & & 1200 & 16.20 & 0.00 \\
\hline & & & & & 1000 & 16.23 & 0.01 \\
\hline & & & & & 801 & 16.23 & 0.01 \\
\hline & & & & & 601 & 16.05 & 0.01 \\
\hline & & & & & 500 & 16.00 & 0.02 \\
\hline & & & & & 400 & 16.33 & 0.02 \\
\hline & & & & & 300 & 16.11 & 0.04 \\
\hline & & & & & 200 & 15.89 & 0.14 \\
\hline & & & & & 150 & 15.67 & 0.16 \\
\hline & & & & & 99 & 15.37 & 0.32 \\
\hline & & & & & 50 & 11.82 & 0.31 \\
\hline & & & & & 4 & & 0.00 \\
\hline 69 & 3 & 55.842 & -48.093 & $22 / 06 / 2014$ & 200 & & \\
\hline & & & & & 150 & & \\
\hline & & & & & 100 & & \\
\hline & & & & & 80 & & \\
\hline & & & & & 60 & & \\
\hline & & & & & 50 & & \\
\hline & & & & & 40 & & \\
\hline & & & & & 30 & & \\
\hline & & & & & 22 & & \\
\hline & & & & & 14 & & \\
\hline & & & & & 10 & & \\
\hline & & & & & 3 & & \\
\hline 70 & 1 & 54.767 & -48.763 & $24 / 06 / 2014$ & 3773 & 14.69 & 0.01 \\
\hline & & & & & 3500 & 15.76 & 0.00 \\
\hline & & & & & 3251 & 16.44 & 0.00 \\
\hline & & & & & 3000 & 16.87 & 0.00 \\
\hline & & & & & 2500 & 16.90 & 0.00 \\
\hline & & & & & 2250 & 16.98 & 0.00 \\
\hline & & & & & 2000 & 17.29 & 0.00 \\
\hline & & & & & 1801 & 17.32 & 0.01 \\
\hline & & & & & 1600 & 17.36 & 0.01 \\
\hline & & & & & 1399 & 17.27 & 0.01 \\
\hline & & & & & 1200 & 16.73 & 0.00 \\
\hline & & & & & 1000 & 16.42 & 0.01 \\
\hline & & & & & 900 & 16.35 & 0.00 \\
\hline & & & & & 800 & 16.23 & 0.01 \\
\hline & & & & & 699 & 16.32 & 0.02 \\
\hline & & & & & 600 & 16.21 & 0.01 \\
\hline & & & & & 499 & 16.16 & 0.05 \\
\hline & & & & & 399 & 16.31 & 0.01 \\
\hline & & & & & 300 & 15.99 & 0.03 \\
\hline & & & & & 200 & 16.01 & 0.10 \\
\hline & & & & & 149 & 15.73 & \\
\hline & & & & & 100 & 14.92 & 0.21 \\
\hline & & & & & 39 & 7.23 & 0.50 \\
\hline & & & & & 4 & 4.16 & 0.32 \\
\hline 71 & 1 & 53.692 & -49.433 & $24 / 06 / 2014$ & 3756 & 14.65 & 0.01 \\
\hline & & & & & 3499 & 15.67 & 0.00 \\
\hline & & & & & 3240 & 16.35 & 0.00 \\
\hline & & & & & 3000 & 16.54 & 0.00 \\
\hline & & & & & 2750 & 16.79 & 0.00 \\
\hline & & & & & 2500 & 16.75 & 0.00 \\
\hline & & & & & 2249 & 17.00 & 0.01 \\
\hline & & & & & 2000 & 17.24 & 0.00 \\
\hline & & & & & 1799 & 17.24 & 0.01 \\
\hline & & & & & 1600 & 17.35 & 0.00 \\
\hline
\end{tabular}




\begin{tabular}{|c|c|c|c|c|c|c|c|}
\hline & & & & & 1399 & 17.23 & 0.01 \\
\hline & & & & & 1200 & 16.67 & 0.01 \\
\hline & & & & & 1000 & 16.23 & 0.01 \\
\hline & & & & & 801 & 16.16 & 0.01 \\
\hline & & & & & 700 & 16.24 & 0.01 \\
\hline & & & & & 600 & 16.20 & 0.01 \\
\hline & & & & & 500 & 16.10 & 0.02 \\
\hline & & & & & 400 & 16.27 & 0.02 \\
\hline & & & & & 350 & 16.12 & 0.01 \\
\hline & & & & & 250 & 15.27 & 0.03 \\
\hline & & & & & 150 & 13.54 & 0.00 \\
\hline & & & & & 100 & 14.58 & 0.15 \\
\hline & & & & & 50 & 8.24 & 0.00 \\
\hline & & & & & 5 & 1.80 & 0.15 \\
\hline 71 & 3 & 53.692 & -49.433 & $24 / 06 / 2014$ & 200 & & \\
\hline & & & & & 150 & & \\
\hline & & & & & 100 & & \\
\hline & & & & & 80 & & \\
\hline & & & & & 70 & & \\
\hline & & & & & 58 & & \\
\hline & & & & & 50 & & \\
\hline & & & & & 40 & & \\
\hline & & & & & 30 & & \\
\hline & & & & & 20 & & \\
\hline & & & & & 10 & & \\
\hline & & & & & 4 & & \\
\hline 72 & 1 & 53.142 & -50.862 & $25 / 06 / 2014$ & 2920 & 14.72 & 0.00 \\
\hline & & & & & 2632 & 15.98 & 0.00 \\
\hline & & & & & 2550 & 15.78 & 0.00 \\
\hline & & & & & 2450 & 16.08 & 0.00 \\
\hline & & & & & 2252 & 16.29 & 0.00 \\
\hline & & & & & 2001 & 16.43 & 0.00 \\
\hline & & & & & 1800 & 16.59 & 0.00 \\
\hline & & & & & 1600 & 16.69 & 0.00 \\
\hline & & & & & 1400 & 16.94 & 0.00 \\
\hline & & & & & 1200 & 17.11 & 0.00 \\
\hline & & & & & 1001 & 16.24 & 0.00 \\
\hline & & & & & 900 & 16.82 & 0.00 \\
\hline & & & & & 800 & 16.19 & 0.00 \\
\hline & & & & & 700 & 16.19 & 0.00 \\
\hline & & & & & 600 & 16.14 & 0.00 \\
\hline & & & & & 500 & 16.03 & 0.01 \\
\hline & & & & & 400 & 15.95 & 0.02 \\
\hline & & & & & 300 & 16.03 & 0.01 \\
\hline & & & & & 201 & 15.71 & 0.01 \\
\hline & & & & & 150 & 15.68 & 0.02 \\
\hline & & & & & 100 & 15.45 & \\
\hline & & & & & 50 & 13.70 & 0.13 \\
\hline & & & & & 25 & 3.39 & \\
\hline & & & & & 5 & 0.67 & 0.15 \\
\hline 73 & 1 & 52.933 & -51.391 & $25 / 06 / 2014$ & 2075 & 16.67 & 0.02 \\
\hline & & & & & 1901 & 16.85 & 0.02 \\
\hline & & & & & 1799 & 16.94 & 0.01 \\
\hline & & & & & 1700 & 17.02 & 0.01 \\
\hline & & & & & 1600 & 17.13 & 0.01 \\
\hline & & & & & 1500 & 17.17 & 0.02 \\
\hline & & & & & 1401 & 17.06 & 0.02 \\
\hline & & & & & 1201 & 16.53 & 0.02 \\
\hline & & & & & 1001 & 16.07 & 0.02 \\
\hline & & & & & 900 & 16.19 & 0.01 \\
\hline & & & & & 800 & 16.40 & 0.05 \\
\hline & & & & & 701 & 16.13 & 0.04 \\
\hline & & & & & 600 & 16.14 & 0.04 \\
\hline & & & & & 500 & 15.91 & 0.06 \\
\hline & & & & & 400 & 15.94 & 0.07 \\
\hline & & & & & 301 & 15.86 & 0.08 \\
\hline & & & & & 200 & 15.67 & 0.15 \\
\hline & & & & & 150 & 15.73 & 0.22 \\
\hline & & & & & 100 & 15.27 & \\
\hline & & & & & 50 & 10.70 & \\
\hline & & & & & 4 & 0.04 & 0.01 \\
\hline 74 & 1 & 52.852 & -51.596 & $25 / 06 / 2014$ & 1259 & 16.46 & 0.04 \\
\hline & & & & & 1151 & 16.50 & 0.04 \\
\hline & & & & & 1002 & 16.43 & 0.02 \\
\hline & & & & & 901 & 16.43 & 0.03 \\
\hline & & & & & 699 & 16.28 & 0.04 \\
\hline & & & & & 601 & 16.23 & 0.04 \\
\hline & & & & & 501 & 16.02 & 0.04 \\
\hline & & & & & 401 & 15.88 & 0.10 \\
\hline & & & & & 300 & 15.76 & 0.09 \\
\hline & & & & & 200 & 15.62 & 0.06 \\
\hline & & & & & 151 & 15.35 & 0.17 \\
\hline & & & & & 100 & 14.77 & 0.50 \\
\hline & & & & & 50 & 13.37 & 0.00 \\
\hline & & & & & 4 & 0.10 & 0.02 \\
\hline 75 & 1 & 52.786 & -51.760 & $25 / 06 / 2014$ & 472 & 15.88 & 0.02 \\
\hline & & & & & 401 & 15.93 & 0.02 \\
\hline & & & & & 301 & 15.72 & 0.00 \\
\hline & & & & & 200 & 15.26 & 0.01 \\
\hline & & & & & 150 & 15.00 & 0.00 \\
\hline & & & & & 101 & 13.80 & 0.11 \\
\hline
\end{tabular}




\begin{tabular}{|c|c|c|c|c|c|c|c|c|c|c|c|}
\hline & & & & & 50 & 10.29 & 0.38 & 7.62 & & & \\
\hline & & & & & 2 & 0.01 & 0.00 & 2.47 & & & \\
\hline \multirow[t]{7}{*}{76} & 1 & 52.500 & -52.500 & $25 / 06 / 2014$ & 251 & 15.39 & 0.09 & 9.58 & & & \\
\hline & & & & & 200 & 13.96 & 0.09 & 8.17 & & & \\
\hline & & & & & 150 & 11.96 & 0.16 & 8.06 & & & \\
\hline & & & & & 100 & 9.90 & 0.53 & 7.85 & & & \\
\hline & & & & & 50 & 8.47 & 0.27 & 7.86 & & & \\
\hline & & & & & 25 & 2.30 & 0.04 & 3.97 & & & \\
\hline & & & & & 5 & & 0.00 & 0.25 & & & \\
\hline \multirow[t]{23}{*}{77} & 1 & 53.000 & -51.100 & $25 / 06 / 2014$ & 2531 & 15.66 & 0.01 & 11.82 & 0.01 & & \\
\hline & & & & & 2449 & 16.04 & 0.01 & 12.18 & & & \\
\hline & & & & & 2401 & 15.89 & 0.01 & 12.07 & & & \\
\hline & & & & & 2201 & 16.30 & 0.01 & 12.03 & & & \\
\hline & & & & & 2000 & 16.60 & 0.00 & 11.71 & 0.02 & & \\
\hline & & & & & 1751 & 16.78 & 0.00 & 11.11 & & & \\
\hline & & & & & 1500 & 17.31 & 0.00 & 11.13 & 0.01 & & \\
\hline & & & & & 1302 & 17.23 & 0.01 & 10.57 & & & \\
\hline & & & & & 1202 & 17.09 & 0.01 & 10.14 & & & \\
\hline & & & & & 1001 & 16.42 & 0.01 & 8.68 & 0.02 & & \\
\hline & & & & & 902 & 16.36 & 0.01 & 8.54 & & & \\
\hline & & & & & 802 & 16.24 & 0.01 & 8.47 & & & \\
\hline & & & & & 701 & 16.43 & 0.00 & 8.52 & & & \\
\hline & & & & & 601 & 16.40 & 0.01 & 8.43 & & & \\
\hline & & & & & 502 & 16.30 & 0.03 & 8.38 & 0.02 & & \\
\hline & & & & & 402 & 16.22 & 0.03 & 8.40 & & & \\
\hline & & & & & 300 & 15.65 & 0.24 & 8.20 & & & \\
\hline & & & & & 201 & 15.26 & 0.56 & 8.17 & & & \\
\hline & & & & & 151 & 15.56 & & 8.14 & & & \\
\hline & & & & & 100 & 14.61 & & 8.05 & & & \\
\hline & & & & & 40 & 7.05 & 0.27 & 5.99 & & & \\
\hline & & & & & 24 & 3.23 & 0.13 & 3.59 & & & \\
\hline & & & & & 4 & 0.38 & 0.04 & 1.09 & & & \\
\hline \multirow[t]{12}{*}{77} & 3 & 52.989 & -51.095 & $26 / 06 / 2014$ & 201 & & & & 0.06 & 0.35 & 1.73 \\
\hline & & & & & 150 & & & & 0.05 & 0.41 & 2.16 \\
\hline & & & & & 100 & & & & 0.09 & 0.51 & 2.99 \\
\hline & & & & & 80 & & & & 0.09 & 0.49 & 3.38 \\
\hline & & & & & 60 & & & & 0.53 & 1.03 & 6.31 \\
\hline & & & & & 45 & & & & 1.05 & 2.25 & 13.68 \\
\hline & & & & & 35 & & & & 0.86 & 2.88 & 16.07 \\
\hline & & & & & 25 & & & & 0.94 & 3.66 & 20.06 \\
\hline & & & & & 20 & & & & 0.82 & 3.52 & 18.92 \\
\hline & & & & & 15 & & & & 0.88 & 3.54 & 20.44 \\
\hline & & & & & 10 & & & & 0.86 & 3.47 & 19.51 \\
\hline & & & & & 5 & & & & 0.54 & 4.00 & 23.31 \\
\hline \multirow[t]{13}{*}{78} & 1 & 51.989 & -53.817 & $27 / 06 / 2014$ & 371 & 15.90 & 0.06 & 12.28 & & & \\
\hline & & & & & 299 & 15.72 & 0.04 & 11.96 & & & \\
\hline & & & & & 200 & 12.94 & 0.13 & 8.59 & 0.38 & 0.63 & 3.02 \\
\hline & & & & & 150 & 11.38 & 0.06 & 8.41 & 0.33 & 0.46 & 2.53 \\
\hline & & & & & 100 & 10.06 & 0.07 & 8.50 & 0.38 & 0.52 & 2.70 \\
\hline & & & & & 80 & & & & 0.24 & 0.49 & 2.78 \\
\hline & & & & & 60 & & & & 0.34 & 0.54 & 2.71 \\
\hline & & & & & 45 & 8.12 & 0.10 & 8.76 & 0.41 & 0.98 & 5.58 \\
\hline & & & & & 35 & & & & 2.82 & 5.26 & 31.22 \\
\hline & & & & & 30 & & & & 2.63 & 4.72 & 36.15 \\
\hline & & & & & 20 & & & & 0.15 & 0.75 & 3.40 \\
\hline & & & & & 10 & & & & 0.19 & 0.90 & 4.96 \\
\hline & & & & & 5 & 0.06 & 0.00 & 0.21 & 0.15 & 1.09 & 5.50 \\
\hline
\end{tabular}

